\title{
Measuring quantile dependence and testing directional predictability between Bitcoin, altcoins and traditional financial assets
}

\author{
Shaen Corbet ${ }^{a, b *}$, Paraskevi Katsiampa ${ }^{c}$, Chi Keung Marco Lau ${ }^{d}$ \\ ${ }^{a}$ DCU Business School, Dublin City University, Dublin 9, Ireland \\ ${ }^{b}$ School of Accounting, Finance and Economics, University of Waikato, New Zealand \\ ${ }^{c}$ Sheffield University Management School, The University of Sheffield, Conduit road, Sheffield, S10 1FL, UK \\ ${ }^{d}$ Department of Accountancy, Finance and Economics, Huddersfield Business School, University of Huddersfield, \\ Queensgate, Huddersfield, HD1 3DH, UK \\ *Corresponding Author: shaen.corbet@dcu.ie
}

\begin{abstract}
This paper studies causal relationships and the potential of improving conditional quantile forecasting between Bitcoin and seven altcoin markets as well as between Bitcoin and three mainstream assets, namely gold, oil, and the S\&P500, by applying the Granger-causality in distribution and in quantiles tests. We find significant bidirectional causality between Bitcoin and all altcoins and assets considered in the two distribution tails. An enhanced forecast of Bitcoin price returns is thus derived by conditioning on altcoins or assets and vice versa during extreme market conditions. However, under normal market conditions the results for the centre of the distribution of the Bitcoin price returns conditional on altcoins depend on both the altcoin considered and quantile under investigation. We also find evidence that Bitcoin is not isolated from financial markets, while this developing financial asset is a strong safe-haven for oil and a weak safe-haven for S\&P500, but it cannot be considered as either a weak or strong safe-haven for gold. Our results reveal a more complete relationship between Bitcoin and altcoins as well as financial assets than was previously considered.
\end{abstract}

Keywords: Bitcoin; Cryptocurrency; Granger Causality in Distribution; Quantile Dependence; Directional Predictability; Cross-quantilogram.

\section{Introduction}

The substantial growth in both the price and publicity surrounding cryptocurrencies at large has generated a substantial debate as to the regulatory requirements, the inherent dangers that are sourced within their structure, particularly within the growing number of substantial cases of 
theft, evidence of market manipulation and other types of illegality that have taken place in recent years. Proponents continue to point to the forthcoming advancement of the digital age, that is central to the placement of blockchain in the modern financial ecosystem. Opponents ask for more care and regulatory influence before creating a product that could potentially generate volatility and contagion effects upon unwilling and unsuspecting financial markets. The co-movement of cryptocurrency pricing structures with other more developed financial markets has been covered quite extensively in recent times (see, e.g., Corbet et al. [2018]), however, further analysis of the co-movements of cryptocurrency pricing behaviour has begun to generate evidence of some substantial 'anomalies' (Griffins and Shams [2018]). Although the literature on interdependencies in cryptocurrency markets has emerged (see for example, Ciaian et al. [2018], Katsiampa [2018a,b], Corbet et al. [2020]), little is known about cryptocurrencies' quantile, including tail, dependence as well as about directional predictability between cryptocurrencies. Further, conditional quantile forecasting has been increasingly used in economic and finance applications (Lee and Yang [2014]), since causality may matter in higher moments or in the dependence structure in a joint density. However, to the best of the authors' knowledge, no previous study has tested the Granger-causality in distribution (GCD) or Granger-causality in quantiles (GCQ) to explore a causal relationship between cryptocurrencies at different states of the market.

Consequently, the aim of this study is to investigate causal relationships as well as the potential of improving conditional quantile forecasting between Bitcoin and seven major altcoins, namely Ripple, Ether, Stellar, Litecoin, Monero, Dash, and NEM, by considering the GCD and GCQ tests, not only under normal market conditions but also under extreme market conditions. For comparison purposes, we also consider three financial assets, namely gold, oil, and S\&P500, and compare the results with those for the altcoins. According to the results, although the predictability of the centre of the distribution (i.e., usual performance) of Bitcoin's price returns conditional on altcoins depends on the altcoin and quantile under investigation, none of the commodities or stock considered can be used to improve the forecast of Bitcoin's price returns under normal market conditions. Moreover, Bitcoin can be used to enhance the forecast of the central region of the distribution of the price returns of both of the considered commodities but not of the price returns of S\&P500 or the price returns of any of the altcoins considered. Nonetheless, when investigating the distribution tails, not only an enhanced quantile forecast of the altcoins' price returns is produced by conditioning on Bitcoin, as could have been expected due to fact that most altcoin orders are executed in Bitcoin, but also an enhanced quantile forecast of Bitcoin's price returns is derived by conditioning on an altcoin during both superior and poor performance. Similar results are obtained for both distribution tails of the pair-wise causal relationships between Bitcoin and commodities or S\&P500. We can thus predict both extreme positive and negative movements in altcoin, commodity and stock returns based on Bitcoin as well as forecast both extreme positive and negative price movements in Bitcoin based on the altcoins, commodities and stock index considered. 
In our study, we also employ the latest directional predictability test of Han et al. [2016], which is used in line with the GCD and GCQ tests, in order to investigate whether altcoins or mainstream financial assets can be used to predict Bitcoin's price movements and vice versa. There are a number of advantages in the utilisation of the directional predictability test, in comparison to that of the Granger-causality tests as will be discussed in Section 3. As will be shown, we find evidence of positive predictability from Bitcoin to altcoins as well as from altcoins to Bitcoin when cryptocurrencies are in either bear or bull market. Nevertheless, during bull market Bitcoin's price returns reveal negative predictability on altcoins' price returns, provided that the price returns of altcoins are high, while during bear market Bitcoin's price returns also show negative predictability on altcoins' returns, provided that the price returns of altcoins are relatively low. Similarly, we find that during bull market altcoins reveal negative predictability on Bitcoin's price returns, when the price returns of Bitcoin are high, whereas during bear market altcoins also reveal negative predictability on Bitcoin's returns, when the price returns of Bitcoin are relatively low. It is therefore shown that the price returns of Bitcoin and altcoins move in the same direction when they both are in bear or bull market but move in opposite directions when Bitcoin price returns are high and altcoin returns are low or when Bitcoin price returns are low and altcoin returns are high. These results hold for all the altcoins considered in this study except for Ether and Monero. With regards to the commodities and stock considered, we find evidence of negative predictability from Bitcoin to the two commodities when both Bitcoin and gold/oil are in either bear or bull market but positive predictability from Bitcoin to S\&P500 when both are in bear market as well as when Bitcoin is in bull market and S\&P500 is either at its median or in bear market. On the other hand, we find positive predictability from gold to Bitcoin when both are in bear market but negative predictability when both are in bull market. We further find negative predictability from oil and S\&P500 to Bitcoin when both oil/S\&P500 and Bitcoin are in either bear or bull markets. Finally, we argue that Bitcoin is a strong safe-haven for oil and a weak safe-haven for S\&P500, but it cannot be considered as either a weak or strong safe-haven for gold.

To the best of the authors' knowledge, this is the first study to thoroughly explore the dependence between Bitcoin and altcoin, commodity or stock returns across the entire range of quantiles using several copula functions and Granger-causality tests in each conditional quantile, with the results providing a more complete overview of the Granger-causality not only in distribution but also in quantiles. This is also the first study to apply the directional predictability test of Han et al. [2016] to test for the predictability of Bitcoin using altcoins or commodities as predictors as well as the predictability of altcoins and commodities while utilising Bitcoin as a predictor, further complementing our analysis. Our results therefore reveal a more complete relationship between Bitcoin and altcoins as well as between Bitcoin and mainstream assets, and illustrate how the relationship changes at different quantiles and at different lags. Our findings are thus of high importance to investors interested in constructing portfolios comprising Bitcoin and altcoins, commodities or stock, 
enabling them to select more appropriate trading strategies.

The rest of the paper is organised as follows: Section 2 reviews relevant academic literature. Section 3 presents the data and methodology employed, while Section 4 discusses the results. Finally, section 5 summarises the key findings of the paper.

\section{Literature review}

The literature on cryptocurrencies has rapidly emerged. One of the most immediate issues for cryptocurrencies at large is the potential existence of an inherent pricing bubble. Corbet et al. [2018] built on the work of Phillips et al. [2011] and Phillips et al. [2015] to examine the existence and dates of potential pricing bubbles in the markets for Bitcoin and Ether presenting evidence that Bitcoin was almost certainly in a bubble phase in late 2017. This echoed the findings of Cheung et al. [2015] who focused on the collapse of Bitcoin's largest exchange, Mt. Gox, to identify numerous short-lived bubbles over the period 2010 through 2014. Cheah and Fry [2015] found that Bitcoin exhibits speculative bubbles with further empirical evidence provided that the fundamental price of Bitcoin is zero, while Fry [2018] introduced a rational bubble model for Bitcoin and other cryptocurrencies that combines both heavy tails and the probability of a complete collapse in asset prices which makes this model a theoretical refinement of the model by Cheah and Fry [2015].

Another topic that has been widely explored in the literature is the volatility of cryptocurrency price returns which has been studied by Katsiampa [2017], Ardia et al. [2018], Phillip et al. [2018], Corbet and Katsiampa [2018], and Baur and Dimpfl [2018], among others, all of whom employed different models to describe cryptocurrencies' volatility. Moreover, Blau [2018] investigated the volatility of Bitcoin across time while testing as to whether the unusual level of its volatility is attributed to speculative trading to find that this speculative trading did not have any relationship with the 2013 price increases nor the dramatic increases in volatility. Furthermore, through the use of a significant database spanning 2010 through 2017, Urquhart [2018] found that realised volatility and the volume of Bitcoin traded, controlled for Bitcoin fundamentals, are both significant drivers of the next day's attention for Bitcoin. On the other hand, Baek and Elbeck [2015] used the S\&P500 to examine relative volatility with Bitcoin using de-trended ratios to find that Bitcoin is internally driven by buyers and sellers, therefore concluding that the Bitcoin market is highly speculative.

Cryptocurrency and other market interconnectedness is another topic of substantial interest when identifying issues within the structures of these new financial assets. Using spanning tests, Brière et al. [2015] found that Bitcoin investments offer significant diversification benefits and showed that the inclusion of even a small proportion of Bitcoins may dramatically improve the risk-return trade-off of well-diversified portfolios. Corbet et al. [2018a] added further support while identifying the relationships between three popular cryptocurrencies and a variety of other financial assets by finding evidence of the relative isolation of cryptocurrencies from the financial and economic assets. Moreover, Baur et al. [2018] analysed the statistical properties of Bitcoin to find that 
it is uncorrelated with traditional asset classes in periods of financial turmoil, while transaction data of Bitcoin accounts showed that Bitcoins are mainly used as a speculative investment and not as an alternative currency or medium of exchange. Dyhrberg [2016a] found several similarities to gold and the US dollar, indicative of hedging capabilities and advantages as a medium of exchange, and that Bitcoin has a place in financial markets and can be classified as something in between gold and the US dollar on a scale from pure medium of exchange advantages to pure store of value advantages. In an attempt to replicate the above findings, Baur et al. [2017] demonstrated that exact replication is not possible and that alternative statistical methodologies provide more reliable, however, very different results, with the findings showing that Bitcoin exhibits distinctively different return, volatility, and correlation characteristics compared to other assets including gold and the United States dollar. Furthermore, Dyhrberg [2016b] showed that Bitcoin can be used as a hedge against stocks in the Financial Times Stock Exchange Index and against the US dollar in the short-term and was thereby found to possess some of the same hedging abilities as gold and can be thus included in the variety of tools available to market analysts to hedge market-specific risk.

Nevertheless, interdependencies within cryptocurrency markets continue to remain relatively under-explored. Among the few studies of interconnectedness in cryptocurrency markets are those of Ciaian et al. [2018], Corbet et al. [2018a], Katsiampa [2018a,b] and Katsiampa et al. [2019a,b]. More specifically, Ciaian et al. [2018] studied interdependencies between Bitcoin and altcoin markets in the short and long-run using the Autoregressive Distributed Lag (ARDL) model and found that the markets are interdependent, with the interdependencies being significantly stronger in the short-run. On the other hand, Corbet et al. [2018a,b], Katsiampa [2018a,b] and Katsiampa et al. [2019a,b] all studied conditional correlations, volatility co-movements and volatility spillovers between cryptocurrencies. However, little is known about cryptocurrencies' quantile, including tail, dependence. Although the Granger-causality in distribution test has found several applications in economics and finance, to date, no previous study has tested the causality in distribution in cryptocurrency markets to explore a causal relationship between cryptocurrencies, even though causality may matter in higher moments or in the dependence structure in a joint density. In cryptocurrency markets, the copula-based GCD test has been previously employed by Dastgir et al. [2019], who studied the causal relationship between Google Trends search queries for Bitcoin and Bitcoin returns and found bidirectional causality which primarily exists in the two tails of the distribution, and Bouri et al. [2019], who found that the global financial stress index strongly Granger-causes Bitcoin returns at the left and right tail of the distribution of the Bitcoin returns conditional on the global financial stress index. Other evidence of similar usage is relatively sparse. Our research therefore builds on the use of GCD testing to examine causal relationships between cryptocurrencies as well as between Bitcoin and other financial assets.

In addition, no previous study has applied the cross-quantilogram to cryptocurrencies in order to study cryptocurrency predictability either. Linton and Whang [2007] were amongst the first to 
introduce a quantilogram in an effort to measure predictability in different parts of a distribution of a stationary time series based on a correlogram of quantile hits. The authors applied this technique to test the hypothesis that a given time series has no directional predictability with a null hypothesis based explicitly on the fact that the past information set of the stationary time series does not improve the prediction that the same time series will be above or below the unconditional quantile. Later, Han et al. [2016] developed on this work to present a directional predictability test in order to measure the quantile dependence between two time series, applying it to US stock returns. The cross-quantilogram of Han et al. [2016] was later adopted by Jiang et al. [2018], who examined the rolling return, intraday, overnight, and daily spillovers of four major agricultural commodities, namely sugar, corn, wheat, and soybeans, involving Chinese and US futures markets. The empirical model was deemed to be very useful by the authors as it captured the extreme quantiles dependence between markets. Other uses of the quantilogram include that of White et al. [2015], who investigated the safe haven properties of gold relative to US stock market sector indices using the bi-variate cross-quantilogram of Han et al. [2016]. The authors split the sample into pre- and post-crisis periods, with results showing that the safe haven properties of gold have a changing nature. Before and after the financial crisis, the authors identify only limited quantile dependence and that gold can be considered a safe haven for most of the sectors, except industrials. Baumöhl and Lyócsa [2017] proposed methods for the estimation and inference in multivariate, multi-quantile models and construct impulse-response functions for the quantiles of a sample of 230 financial institutions around the world and study how financial institution-specific and system-wide shocks are absorbed by the system. The authors showed how the long-run risk of the largest and most leveraged financial institutions is very sensitive to market wide shocks in situations of financial distress, suggesting that the methodology can prove a valuable addition to the traditional toolkit of policy makers and supervisors. Further, the cross-quantilogram has several advantages compared to other test statistics for directional predictability, as it is primarily conceptually appealing and relatively simple to interpret, while not requiring moment conditions like the ordinary correlogram and other statistics that are derived from within. Consequently, our research also builds on the use of the cross-quantilogram of Han et al. [2016] in order to study directional predictability between Bitcoin and altcoins as well as between Bitcoin and financial assets. To the best of the authors' knowledge, the cross-quantilogram has been applied to cryptocurrencies only in the studies of Bouri et al. [2019] on the relationship between the global financial stress index and Bitcoin, in which the authors found only limited directional predictability from the global financial stress index to Bitcoin returns in the medium term, and of Shahzad et al. [2019] on the relationship between Bitcoin with financial indices, in which the authors found that Bitcoin cannot be regarded as a strong safe-haven for any of the stock indices considered. This is therefore the first study applying the cross-quantilogram to explore the relationship of cryptocurrencies. 


\section{Data and methodology}

\subsection{Data}

Our dataset consists of daily closing prices for cryptocurrencies that have been in existence for over three years and had a market capitalisation exceeding $\$ 500 \mathrm{~m}$ as of January 2019. Consequently, our dataset comprises of daily figures for eight major cryptocurrencies, namely Bitcoin, Ripple, Ether, Stellar, Litecoin, Monero, Dash, and NEM, from 7 August 2015 (as the earliest date available for Ether) to 20 January 2019, resulting in 1,263 observations for each cryptocurrency. The prices are listed in US Dollars and the data are sourced at https://coinmarketcap.com/coins/. Data for gold prices are collected from Thompson Reuters DataStream, while data for the West Texas Intermediate crude oil and S\&P500 are sourced from Bloomberg for the same period as the selected cryptocurrencies. In our analysis, we use the logarithmic price returns.

\subsection{Granger-causality in distribution (GCD) and Granger-causality in quantiles (GCQ) tests}

In this study, we follow the methodology of Lee and Yang [2014] for GCD testing and for investigating the dependence between Bitcoin and the other variables of our interest. The GCD test is preferred to other methods such as the linear Granger causality test due to its ability to capture the asymmetric dependence in the different quantiles between two variables that could potentially exist. Furthermore, in terms of modelling, there is an enhancement with the use of the GCD test for exploration of the relationship between Bitcoin and the other variables under consideration, as this models the causal relationship at the boundaries of the return distributions instead of the centre alone. Consequently, following Lee and Yang [2014], we apply a parametric copulabased approach to model the dependence structure between Bitcoin and altcoins as well as between Bitcoin and financial assets using six copula density functions, namely the Gaussian, Frank, Clayton, Clayton Survival, Gumbel, and Gumbel Survival copula. These copula functions represent different dependence structures. We therefore construct a test for GCD which is parametric. Noting that different parametric copula functions imply different dependence structures, we design a method to compare them in an entropy with the independent copula density. The test thus compares the outof-sample predictive ability of the copula functions relative to the benchmark independent copula density.

The proposed GCD out-of-sample test is applied in line with the test of Hong and Li [2004], in which the null hypothesis states that: $H_{0}: c(u, v)=1$, where $c(u, v)$ represents the conditional copula density function, using $u$ and $v$ as the conditional probability integral transforms of two variables, $X_{t}$ and $Y_{t}$, respectively. In other words, under the null hypothesis $X_{t}$ does not Granger

cause $Y_{t}$ in distribution. The predicted conditional variance for $X_{t}$ and $Y_{t}, \hat{h}_{x, t+1}$ and $\hat{h}_{y, t+1}$, respectively, are calculated by:

$$
\hat{h}_{x, t+1}=\hat{\beta}_{x 0}+\hat{\beta}_{x 1} x_{t}^{2}+\hat{\beta}_{x 2} \hat{h}_{t, x}
$$




$$
\hat{h}_{y, t+1}=\hat{\beta}_{y 0}+\hat{\beta}_{y 1} y_{t}^{2}+\hat{\beta}_{y 2} \hat{h}_{t, x}
$$

Computation of the CDF values of $\hat{u}_{t+1}$ and $\hat{v}_{t+1}$ for $x_{t+1}$ and $y_{t+1}$ are carried out by the empirical distribution function (EDF), where the estimation of a non-parametric copula function is pared EDF values $\left(\hat{u}_{t+1}, \hat{v}_{t+1}\right)_{t=R}^{T-1}$ by means of the following specified quartic kernel function:

$$
k(u)=\frac{15}{16}\left(1-u^{2}\right)^{2} I(|u|) \leq 1
$$

In our study, the outcome of the GCD utilising the test statistic of Hong and Li [2004] for $\left(\hat{u}_{t+1}, \hat{v}_{t+1}\right)_{t=R}^{T-1}$ is found significant at the $1 \%$ level for all pairs between Bitcoin and altcoin, commodity, or stock returns (see Appendix, Table A1), which implies the significance of the GCD between Bitcoin and altcoins, gold, oil and S\&P500. Nevertheless, significant GCD does not imply Granger-causality in each conditional quantile. Moreover, the GCD test result does not inform us about which specific conditional quantiles result in GCD, although it is frequently useful to know which quantile leads to the GCD, especially in portfolio and risk management. Value-at-Risk (VaR) in particular is a quantile in tail that is widely used in capital budgeting and risk control. Since the aim of this study is to explore the potential of improving quantile forecasting of Bitcoin using information from altcoins and financial assets and vice versa, it is therefore of great importance to further investigate which specific quantile leads to GCD. Consequently, following Lee and Yang [2014], next we define Granger-causality in quantile (GCQ), for which quantile forecasts are computed from inverting a conditional copula distribution, and develop a test for GCQ, focusing on three distinct regions of the distribution, namely the right tail (90\% quantile, $95 \%$ quantile and $99 \%$ quantile), the central region ( $40 \%$ quantile, median and $60 \%$ quantile), and the left tail (1\% quantile, $5 \%$ quantile and $10 \%$ quantile). We therefore aim to forecast the conditional quantile, $q_{\alpha}\left(Y_{t} \mid F_{t}\right)$, where $\alpha$ is the left tail probability with $q_{\alpha}\left(Y_{t} \mid F_{t}\right)$ being the conditional quantile that is obtained by the inverse function of a conditional distribution function:

$$
q_{\alpha}\left(Y_{t} \mid F_{t}\right)=F_{Y}^{-1}\left(\alpha \mid F_{t}\right)
$$

where $F_{Y}\left(Y_{t} \mid F_{t}\right)$ is shown as the predicted conditional distribution function of $Y_{t}$. We compute the inverse as $q\left(Y_{t} \mid F_{t}\right)$ from:

$$
\int_{-\infty}^{q_{\alpha}\left(Y_{t} \mid F_{t}\right)} f \gamma\left(y \mid F_{t}\right) d y=\alpha
$$

where $f \gamma\left(y \mid F_{t}\right)$ is the predicted conditional distribution function. The forecasting quantile models $q_{\alpha}\left(Y_{t} \mid F_{t}\right)$ are calculated by the solution of the equation

$$
C_{u}\left(F_{x}\left(x_{t+1}\right), F_{Y}\left(q_{\alpha}\left(Y_{t} \mid F_{t}\right)\right)\right)=\alpha
$$


The evaluation of the ability to predict the forecasting quantile models $q_{\alpha}\left(Y_{t} \mid F_{t}\right)$ is derived from the seven copula functions (including the benchmark independent copula) for $C(u ; v)$, where the 'check' loss function of Koenker and Bassett Jr [1978] is used ${ }^{1}$. The expected check loss for a quantile forecast $q_{\alpha}\left(Y_{t} \mid F_{t}\right)$ at a given left tail probability $\alpha$ is:

$$
Q(\alpha)=E\left[\alpha-I\left(Y_{t}-q_{\alpha}\left(Y_{t} \mid F_{t}\right)<0\right)\right]\left(Y_{t}-q_{\alpha}\left(Y_{t} \mid F_{t}\right)\right)
$$

where the $k^{t h}$ type of copula function is denoted as $C_{k}(u, v)(k=1, \ldots, 7)$. Every individual copula distribution function $C_{k}(u, v)$, is denoted by the equivalent quantile forecast of $q_{, k}\left(Y_{t} F_{t}\right)$ and the check loss expected as $Q_{k}(\alpha)$. To compare the benchmark copula (i.e., the independent copula representing model 1$)$ and model $k(=2, \ldots, 7)$, we consider the equivalent check loss-differential:

$$
D_{k}=Q_{1}(\alpha)-Q_{k}(\alpha)
$$

which is estimated by:

$$
\hat{D}_{k, p}=\hat{Q}_{1, p}(\alpha)-\hat{Q}_{k, p}(\alpha)
$$

where

$$
\hat{Q}_{k, p}(\alpha)=\frac{1}{p} \sum_{t=R}^{T-1}\left[\alpha-I\left(Y_{t}-q_{\alpha\left(Y_{t} \mid F_{t}\right)}\right)<0\right]\left(Y_{t}-q_{\alpha}\left(Y_{t} \mid F_{t}\right)\right), \text { where } k=1, \ldots, 7
$$

The conditional quantile forecasts from using the copula distribution function $C_{k}(k=2, \ldots, 7)$ with the largest value $\hat{D}_{k, p}$ will be preferred. To statistically compare the conditional quantile forecast $q_{\alpha, k}\left(Y_{t} \mid F_{t}\right)$, we therefore test for GCQ based on the multiple parametric copula functions. In particular, we test the null hypothesis $H_{0}: E\left(D_{k}\right) 0,(k=2, \ldots, 7)$, indicating NGCQ, where none of the conditional quantile forecasts computed from copula $C_{k}(k=2, \ldots, 7)$ is better than the benchmark quantile forecast computed from the independent copula distribution, $C_{1}$. For more information, see Lee and Yang [2014].

It is worth noting that the GCQ test also has several advantages compared to other techniques investigating causality. The first generation of causality tests is linear in nature testing the null hypothesis that $Y_{t}$ does not Granger cause $X_{t}$ if the lags of $Y_{t}$ do not introduce additional contribution to the forecasting performance of $X_{t}$ (Granger [1969]). Such tests, however, are sensitive to deviations from the assumption that the error term is normally distributed (i.e., using conditional mean regression models in which the causal relations are linear), and we know that relationships between financial variables could be non-linear, especially for high-frequency data (Kumar [2017]).

\footnotetext{
${ }^{1}$ The reader is referred to the study of Lee and Yang [2014] for detailed information.
} 
Brock et al. [1996] (BDS) test can be used for testing against a variety of possible deviations from independence including linear dependence and non-linear dependence. As the Data Generating Process (DGP) of a time series may be deviated from the normally distributed assumption, Diks and Panchenko [2006] designed a non-parametric test for non-linear Granger causality ${ }^{2}$. However, the nature of non-linear components in a time series is usually unknown, and it is crucial to capture the empirical features observed in the empirical data. In this regard, we need to apply causality analysis which is not only focusing on the first moment but also on higher moments, skewness, kurtosis, and quantiles. The conventional non-linear Granger causality test only looks at the first and second moments of an unknown DGP (for example, jump, structural breaks in mean and trend), while our study investigates dependence structure between two time series relying on a wide range of quantiles using several copula functions and Granger-causality tests in each conditional quantile.

Another stream of studies look at the spillover indices of Diebold and Yilmaz [2009, 2012], Diebold and Yilmaz [2014], which are popular methods for measuring total interdependence or 'connectedness' in a dynamic system of random variables. The notion of 'net receiver of spillovers' and 'net transmitter of spillovers' may be perceived as a kind of causality. However, these tests cannot model the joint distribution of simultaneous events. Our test of GCQ applies parametric copula functions, which capture different non-linear dependence structures, and this method was implemented by comparing different non-linear dependence structures in an entropy with the independent copula density. Copulas constitute a mathematical tool for modelling the joint distribution of simultaneous events. The GCQ test actually evaluates non-linear causalities and possible causal relations in 'distribution", and it relies on the boundary-modified kernel function used by Hong and Li [2004]. Therefore, the proposed test captures the existence of uncaptured non-linearity. This approach therefore allows not only for examining Granger causality in quantiles. Bitcoin's market is very volatile and we need to apply a tail risk approach in this market (Shahzad et al. [2019]). Therefore, the suitability of applying a quantile-based approach is justified and we need to be able to assess the predictability of Bitcoin returns in specific quantile scenarios based on the various quantiles of Bitcoin-Altcoin and Bitcoin-financial asset.

\subsection{Directional predictability test}

In this study, in line with the Granger-causality tests discussed above, we further employ the directional predictability test of Han et al. [2016], the cross-quantilogram, in order to investigate whether altcoins or other financial assets can be utilised to predict changes in Bitcoin's price returns and vice versa. Consequently, the cross-quantilogram enables us assess the forecasted performance

\footnotetext{
${ }^{2}$ Other non-linear Granger causality tests include Hill [2007]. However, these tests do not allow the testing of Granger causality from the upper (lower) tails of one distribution to the lower (upper) tails of another distribution, while tail risk spillovers across assets is obvious.

${ }^{3}$ The distribution is non-linear in its nature.
} 
of Bitcoin while utilising an altcoin or a financial asset as a predictor and, conversely, we can assess the forecasted performance of altcoins and financial assets while utilising Bitcoin as a predictor. The cross-quantilogram has several advantages over the GCQ test and other alternative methods. First of all, one of its most important advantages is its capability to spontaneously detect the direction, duration, and magnitude of the relationship between two variables, and can thus provide beneficial information for the trading strategies employed by investors. Secondly, the directional predictability test enables selection of arbitrary quantiles by researchers, as opposed to the GCD test which uses pre-set quantiles. Thirdly, the utilisation of large lags in the directional predictability test is allowed by the usage of the bootstrap technique. Additionally, researchers are able to consider lengthy lags in comparison with regression type methods (Engle and Manganelli [2004]). A further advantage of using the cross-quantilogram is that it is primarily conceptually appealing and simple to interpret. Finally, since the method is based on quantile hits, it does not require moment conditions like the ordinary correlogram and statistics like the variance ratio that are derived from it.

The cross-quantilogram is defined as the cross-correlation of the quantile-hit (or quantile-exceedance) process and captures serial dependence between two series at different conditional quantile levels. It therefore provides a quantile-to-quantile relationship for the predictability from one variable to another and can thus be used to examine the quantile-to-quantile relationship between Bitcoin and altcoins or financial assets. The linear regression equation for the conditional quantile of a variable is stated as:

$$
q_{\alpha\left(y_{t+1} \mid F_{t}\right)}=\beta_{0, \alpha}+\beta_{1, \alpha} x_{t}+\beta_{2, \alpha} x_{t} q_{\alpha}\left(y_{t} \mid F_{t-1}\right)+\beta_{3, \alpha}\left|y_{t}\right|
$$

where $x_{t}$ and $y_{t}$ are the predictor and predicted variable, respectively, and $q_{\alpha\left(\tau_{t+1} \mid F_{t}\right)}$ is the conditional quantile of the predicted variable given the information $F_{t}$ at time $t$. For detection of the directional predictability, there is a provision in the figure for the cross-quantilogram $\hat{p}_{\alpha}(k)$. We test the null hypothesis that an altcoin or financial asset has no directional predictability for Bitcoin, and vice versa that Bitcoin has no directional predictability for altcoins or assets.

The sample cross-quantilogram $\hat{p}_{\alpha}(k)$ is defined as:

$$
\hat{p}_{\alpha}(k)=\frac{\sum_{t=k+1}^{T} \Psi_{\tau_{1}}\left(y_{1, t}-\hat{q}_{1, t}\left(\tau_{1}\right)\right) \Psi_{\tau_{2}}\left(y_{2, t-k}-\hat{q}_{2, t-k}\left(\tau_{2}\right)\right)}{\sqrt{\sum_{t=k+1}^{T} \Psi_{\tau_{1}}^{2}\left(y_{1, t}-\hat{q}_{1, t}\left(\tau_{1}\right)\right)} \sqrt{\sum_{t=k+1}^{T} \Psi_{\tau_{2}}^{2}\left(y_{2, t-k}-\hat{q}_{2, t-k}\left(\tau_{2}\right)\right)}}
$$

for $k=0,1,2, \ldots$, where $\hat{q}_{i, t}\left(\tau_{i}\right)=x_{i t}^{T} \hat{\beta}_{i}\left(\tau_{i}\right)$, while $y_{t}$ and $x_{t}$ represent the predicted variable and predictor, respectively. The sample cross-quantilogram $\hat{p}_{\alpha}(k)$ measures the directional predictability from one variable to another, as it considers dependence in terms of the direction of deviation from conditional quantiles. The case of no directional predictability is represented by $\hat{p}_{\alpha}(k)=0$, whereas high dependence between two variables is represented by a larger value of $\hat{p}_{\alpha}(k)$.

Following Han et al. [2016], when testing the directional predictability from altcoins and financial 
assets to Bitcoin, we consider a wide range for $\alpha_{1}\left(\alpha_{1}=0.05,0.1,0.2,0.3,0.5,0.7,0.8,0.9\right.$ and 0.95$)$ for the quantiles of Bitcoin, $q_{1}\left(\alpha_{1}\right)$, whereas for the quantiles of the altcoins and assets, $q_{2}\left(\alpha_{2}\right)$, we use $\alpha_{2}=0.1,0.5$, and 0.9. Accordingly, when assessing the directional predictability from Bitcoin to altcoins and assets, we consider a wide range for $\alpha_{1}\left(\alpha_{1}=0.05,0.1,0.2,0.3,0.5,0.7,0.8,0.9\right.$ and $0.95)$ for the quantiles of altcoins and assets, $q_{1}\left(\alpha_{1}\right)$, while we use $\alpha_{2}=0.1,0.5$, and 0.9 for the quantiles of Bitcoin, $q_{2}\left(\alpha_{2}\right)$. In the respective graphs, the maximum lag that is considered is two months (i.e., $\mathrm{k}=60$ days) ${ }^{4}$. Moreover, we depict the $95 \%$ bootstrap confidence interval for no predictability based on 10000 bootstrapped replications while using the non-parametric stationary bootstrap (SB) estimation of Politis and Romano [1994] for estimating the critical values from the limited distribution. The SB is termed as a block bootstrap technique using random lengths of blocks. The SB re-sample is based on the original sample and is strictly stationary ${ }^{5}$.

\section{Empirical findings}

\subsection{Summary statistics}

Table 1 presents summary statistics for the price returns of all the series considered in our study. It can be easily noticed that all cryptocurrencies provide positive average daily returns. More specifically, mean daily returns range from $0.16 \%$ (Litecoin) to $0.47 \%$ (NEM). Moreover, the standard deviation ranges between $3.99 \%$ (Bitcoin) and $8.8 \%$ (NEM), suggesting that more recently launched cryptocurrencies exhibit higher variability. When comparing these results to those for the commodities and stock returns considered in our study, we notice that the average returns of all the three financial series considered (i.e., gold, oil, and S\&P500) are remarkably much lower and in fact very close to zero, while also exhibiting much lower levels of variability, as measured by the standard deviation, ranging from $0.77 \%$ (gold) to $2.33 \%$ (oil). Furthermore, although the price returns of Bitcoin, Ether, and S\&P500 are negatively skewed, indicating that the left tail is longer than the right one for these three, the price returns of all the other cryptocurrencies as well as the two commodities considered in this study are positively skewed.

\section{Insert Table 1 about here}

\subsection{Granger-causality in quantiles $(G C Q)$ test results}

As mentioned in the previous section, in this study, we uncover the causality dynamics between Bitcoin and seven major altcoins as well as gold, oil, and S\&P500, by computing the quantile forecasts that rely on the inversion of the parametric conditional copula distribution. To examine

\footnotetext{
${ }^{4}$ For more information, see Han et al. [2016] and Jiang et al. [2017]

${ }^{5}$ For details of comparison of some common block bootstrap methods, please refer to Lahiri et al. [1999]
} 
the dependence, we used the model of Lee and Yang [2014] which can not only model the potential asymmetric dependence between two variables but can also model the causal relation at the extremes of the return distributions rather than only at the centre. Consequently, in our empirical study, we focused on all the three regions of the distribution: the left tail (1\% quantile, $5 \%$ quantile and $10 \%$ quantile), the central region (40\% quantile, median and $60 \%$ quantile) and the right tail (90\% quantile, 95\% quantile and 99\% quantile), similar to Lee and Yang [2014]. More specifically, we studied pair-wise causal relationships between Bitcoin and altcoins, gold, oil, and S\&P500, by comparing multiple copula functions with the independent copula function, in order to test for GCQ of one market's return conditional on another market's return. We computed the quantile forecasts by inverting the conditional copula distribution, using six copulas, namely the Gaussian, Frank, Clayton, Clayton Survival, Gumbel, and Gumbel Survival copulas, and compared the check loss functions to evaluate predictive ability of different quantile forecasting copula models relative to the quantile forecasts without GCQ. The quantile forecasts without GCQ were computed from the marginal distribution, which is equivalent to the quantile forecast from using the independent copula. Under the null hypothesis of the Reality Check test, none of the six copula functions considered (which model GCQ) makes better quantile forecast than the independent copula (without GCQ).

The quantile causality test results for the causal relationship between the returns of Bitcoin and altcoins or mainstream assets by using the GCQ test as an explorative tool can be found in Tables 2 through 4, where the results of testing for GCQ are reported in p-values. Table 2 in particular presents the results of testing for GCQ from Bitcoin to altcoins. Small p-values of the Reality Check presented suggest the rejection of the null hypothesis of no GCQ in the corresponding quantiles, indicating that an enhanced quantile forecast of the altcoin price returns is produced by conditioning on Bitcoin at several quantiles. More specifically, based on the p-values of the GCQ test, the price returns of Bitcoin strongly Granger-cause the price returns of any of the seven altcoins considered at the $90 \%, 95 \%$, and $99 \%$ quantiles and hence at the right tail of the distribution of any altcoin's return conditional on the returns of Bitcoin (i.e., during superior performance). Consequently, causality is observed at times of bullish markets and it is therefore possible to predict extreme positive movements in altcoin price returns based on Bitcoin price movements. We further notice that Bitcoin's price returns strongly Granger-cause the price returns of all the altcoins at any other investigated quantile of the right tail (i.e., $60 \%, 70 \%$, and $80 \%$ ) as well. Similar results are found for the $5 \%$, and $1 \%$ quantiles and hence for the left tail of the distribution of any altcoin's return conditional on the returns of Bitcoin (i.e., during extremely poor performance), although for Litecoin we can reject the null hypothesis of no GCQ at the 5\% quantile only at the $10 \%$ level of significance, indicating that Bitcoin's price returns also strongly Granger-cause the price returns of altcoins at the left tail. Consequently, it is also possible to predict extreme negative movements in altcoin's returns based on Bitcoin's price movements. It is worth noting, though, that the p-values 
at the $10 \%$ quantile are higher, but when using the $10 \%$ level of significance, we fail to reject the null hypothesis of no GCQ only for Litecoin. Furthermore, when investigating the remaining left tail quantiles (i.e., the $20 \%, 30 \%$, and $40 \%$ quantiles) as well as the $50 \%$ quantile, Bitcoin's price returns do not significantly Granger-cause the price return of any altcoin, and therefore we cannot predict altcoins' price movements based on Bitcoin's price movements in such cases.

\section{Insert Table 2 about here}

On the other hand, Table 3 reports the result of causality from altcoins to Bitcoin. Accordingly, small p-values of the GCQ test suggest the rejection of the null hypothesis of no GCQ, indicating that an enhanced quantile forecast of the Bitcoin price returns can be obtained by conditioning on an altcoin. More specifically, the p-values at the $90 \%, 95 \%$, and $99 \%$ quantiles suggest that the price returns of any of the altcoins considered in our study strongly Granger-cause the price returns of Bitcoin at the right tail of the distribution (i.e., during superior performance). Therefore, it is possible to predict Bitcoin's extreme positive price movements based on the seven altcoins considered. It can also be noted that the altcoins' price returns strongly Granger-cause the price returns of Bitcoin at any other investigated quantile of the right distribution tail (i.e., $70 \%$ and $80 \%$ ) as well. Similar results are displayed for the $1 \%$ and $5 \%$ quantiles and thus for the left tail of the distribution of the Bitcoin return conditional on the returns of each of the seven altcoins considered

(i.e., during extremely poor performance). Therefore, it is also possible to predict extreme negative movements in Bitcoin's price returns based on the seven major altcoins considered. Nevertheless, when inspecting the $10 \%$ quantile, only the price returns of Stellar Granger-cause the price returns of Bitcoin at the $1 \%$ level of significance, while the price returns of Ripple and Litecoin Grangercause the price returns of Bitcoin at a $5 \%$ significance level. Interestingly, the price returns of Ether, Monero, and Dash do not Granger-cause the price returns of Bitcoin at the $10 \%$ quantile under any conventional level of significance. Moreover, when investigating the $20 \%$ and $30 \%$ quantiles, only the price returns of Litecoin significantly Granger-cause Bitcoin's price returns. Finally, when exploring the central region, we notice that the price returns of all the altcoins strongly Granger-cause the price returns of Bitcoin at the $60 \%$ quantile. However, only some of the altcoins Granger-cause the price returns of Bitcoin at the $40 \%$ and $50 \%$ quantiles. The Granger-causality in distribution test results for the centre of the distribution of the Bitcoin price returns conditional on altcoins therefore depend on both the altcoin considered and quantile under investigation.

\section{Insert Table 3 about here}

Finally, Table 4 presents the results of testing GCQ from Bitcoin to gold, oil, and S\&P500, and vice versa. Interestingly, we find similar results to those reported for the pair-wise causal 
relationships between Bitcoin and altcoins in the distribution tails. More specifically, the nearly zero p-values presented for both the right (99\%, 95\%, and $99 \%$ quantiles) and left (1\%, 5\%, and $10 \%$ quantiles) distribution tails, which correspond to superior and poor performance, respectively, suggest the rejection of the null hypothesis of no GCQ. Therefore, an enhanced quantile forecast of the Bitcoin price returns is produced by conditioning on gold, oil, and S\&P500, and the other way around, i.e., an enhanced quantile forecast of the gold, oil, and S\&P500 price returns can be derived by conditioning on Bitcoin. Similar results are also found for all the remaining quantiles except for the $50 \%$ quantile for any pair-wise causal relationship between Bitcoin and mainstream assets. The only exception to this emerges from the causality results from Bitcoin to S\&P500, for which we cannot reject the null hypothesis of no GCQ at the $20 \%, 30 \%, 60 \%$, and $80 \%$ quantiles either, indicating that we cannot derive an enhanced quantile forecast of the S\&P500 price returns by conditioning on Bitcoin for these quantiles.

\section{Insert Table 4 about here}

Consequently, although the predictability of the central region of the distribution (50\% quantile, usual performance) of Bitcoin's price returns conditional on altcoins depends on the altcoin under investigation, none of the commodities or stock considered can be used to improve the $50 \%$ quantile forecast of Bitcoin. Moreover, Bitcoin can be used to improve the $50 \%$ quantile forecast only of the considered commodities but not the price return of S\&P500 or the return of any altcoin. Nonetheless, when investigating the distribution tails, not only an enhanced quantile forecast of the altcoin price returns is produced by conditioning on Bitcoin, as could have been expected due to fact that most altcoin orders are executed in Bitcoin, but also an enhanced quantile forecast of the Bitcoin price returns is derived by conditioning on an altcoin during both superior and poor performance. Similar results are obtained for both distribution tails of the pair-wise causal relationships between Bitcoin and commodities or stock. We can thus predict both extreme positive and negative movements in altcoin, commodity, and stock price returns based on Bitcoin as well as predict both extreme positive and negative movements in Bitcoin price returns based on the altcoins, commodities and stock considered. One explanation for the above findings is that only extreme market conditions lead to significant investors' attention that triggers transmission of information from one market to another, and these phenomena could be resulting from herding behaviour. In addition, such findings of predictability point to return anomaly, thus contradicting the notion of market efficiency. As argued by Baur et al. [2018] and Jiang et al. [2018], it is not surprising to find evidence of inefficiencies in cryptocurrencies, such as Bitcoin, as they are relatively young and volatile, while there is also a lack of a reasonable pricing mechanism as well as irrational behaviour of market participants (Jiang et al. [2018]). Another possible explanation of the results emerges from overconfidence-based models (Thaler [1985]; Hirshleifer [2001]; Daniel and Hirshleifer [2015]). 
As argued by Yeh and Yang [2011], overconfidence increases market volatility and trading volume, two features that highly characterise large cryptocurrencies. In fact, Cheah et al. [2018] indicated that a key driver of the formation of Bitcoin prices is the confidence of its users.

\subsection{Directional predictability test results}

The previous sub-section analysed the relationship between the price returns of Bitcoin and altcoin, gold, oil, and S\&P500 not only at the centre but also at the extremes of the price return distributions and provided confirmation of the hypothesis that causality between the price returns of Bitcoin and altcoins, financial assets and commodities is anticipated at high quantiles. Consequently, it is crucial to conduct complementary analysis at the extreme low and high quantiles to further investigate the directional predictability. Therefore, in this sub-section the results from Han et al.'s [2016] directional predictability test are discussed.

\subsubsection{Directional predictability tests from Bitcoin to altcoins}

The findings of the sample cross-quantilograms $\hat{p}_{\alpha}(k)$ representing directional predictability from Bitcoin to altcoins' price returns are shown in Figures 1 through 7. Panels a and b of each Figure represent the case where the price return of Bitcoin is in the lower quantile, that is, $q_{2}\left(\alpha_{2}\right)$ for $\alpha_{2}=$ 0.1 , and upper quantile, that is, $q_{2}\left(\alpha_{2}\right)$ for $\alpha_{2}=0.9$, respectively. An investigation of the crossquantilograms illustrating the predictability from Bitcoin to the seven altcoins under consideration (Figures 1 through 7 ) reveals that there is a consistent pattern across several cross-quantilograms. More specifically, when Bitcoin's price returns are located at the low quantile of $\alpha_{2}=0.1$, and are thus in bear market, the cross-quantilograms $\hat{p}_{\alpha}(k)$ for $\alpha_{1}=0.05,0.1,0.2$, and 0.3 are positive and significant at most lags. This finding indicates overall positive directional predictability from Bitcoin to altcoins when both Bitcoin and altcoins are in low quantiles, suggesting that when Bitcoin's price changes are negative and of very large magnitude, it is very likely that altcoins will also display very large negative price changes in the following days. In contrast, the cross-quantilograms $\hat{p}_{\alpha}(k)$ for $\alpha_{1}=0.7,0.8,0.9$, and 0.95, when Bitcoin's returns are at the low quantile, are negative and significant at most lags. This result suggests that when Bitcoin's price changes are very low, it is very likely that altcoins will experience very large positive changes in the following days. The above results hold for all the considered altcoins except for Ether, Monero, and NEM (Panel a of Figures 2,5 , and 7 , respectively), for which the results are rather mixed.

On the other hand, when Bitcoin's returns are located at the upper quantile of $\alpha_{2}=0.9$, and are thus in bull market, the cross-quantilograms $\hat{p}_{\alpha}(k)$ for $\alpha_{1}=0.05,0.1,0.2$, and 0.3 are negative and significant at most lags for all the altcoins. Consequently, when Bitcoin 's return is high, there is an increased likelihood of having a large negative loss in altcoins. Exceptions to this include Ether and Monero (Panel b of Figures 2 and 5, respectively) for which the evidence is rather mixed since the cross-quantilograms show both positive and negative values at different lags. As opposed 
to this result, when Bitcoin's returns are at the high quantile, the cross-quantilogram $\hat{p}_{\alpha}(k)$ for $\alpha_{1}=0.7,0.8,0.9$, and 0.95 is positive and significant indicating that when Bitcoin's return is high, there is increased likelihood of having a large positive gain in altcoins. Once again, this conclusion holds for Ripple, Stellar, Litecoin, and Dash, while the results are rather mixed for Ether and Monero (Panel b of Figures 2 and 5, respectively).

The results in Figures 1 through 7 therefore reveal that Bitcoin's price returns are overall helpful in predicting altcoin's extreme price movements when Bitcoin is in either bear or bull market. However, during bull market Bitcoin's price return reveals negative predictability on altcoins' price returns, provided that the price returns of altcoins are high, whereas during bear markets Bitcoin's return also shows negative predictability on altcoins' returns, provided that the price returns of altcoins are relatively low. The above results hold in particular for Ripple, Stellar, Litecoin, Dash, and NEM, while the results for Ether and Monero are mixed. It is worth noting that the results in the cross-quantilograms for Ether and Monero are also less significant compared to the crossquantilograms for all the other altcoins considered, when Bitcoin is either in the low or high quartile.

It is also worth mentioning that the cross-quantilogram $\hat{p}_{\alpha}(k)$ for $\alpha_{1}=0.5$ is mostly insignificant while showing both positive and negative values for both the low and high quantiles of Bitcoin's returns, irrespective of the altcoin under consideration. This suggests that Bitcoin's price return is not helpful in predicting whether altcoin price returns are located below or above their median. This result is in accordance with the GCQ test results previously discussed.

\section{Insert Figures 1 through 7 about here}

\subsubsection{Directional predictability tests from altcoins to Bitcoin}

Next, the sample cross-quantilograms $\hat{p}_{\alpha}(k)$ representing directional predictability from altcoins to Bitcoin price returns are discussed. These are illustrated in Figures 8 through 14. Accordingly, panels $a$ and $b$ of each Figure exhibit the case where the price return of an altcoin is in the lower quantile, that is, $q_{2}\left(\alpha_{2}\right)$ for $\alpha_{2}=0.1$, and upper quantile, that is, $q_{2}\left(\alpha_{2}\right)$ for $\alpha_{2}=0.9$, respectively. Although several discrepancies are observed in the cross-quantilograms for the different cryptocurrencies regarding the quantiles $q_{1}\left(\alpha_{1}\right)$ for Bitcoin and lag orders for which they are significant, it can be noticed that overall there is a rather consistent pattern across the seven altcoins considered and that the cross-quantilograms are significant at several lags for different values of $\alpha_{1}$. More specifically, based on the sample cross-quantilograms at the low quantile for the different altcoins, we notice that the cross-quantilograms $\hat{p}_{\alpha}(k)$ for $\alpha_{1}<0.5$ are mostly positive and significant at the different lags, suggesting that when an altcoin's return is low, it is less likely to have a large positive gain in Bitcoin, while the cross-quantilograms for $\alpha_{1}>0.5$ are mostly negative, indicating that, when an altcoin's return is low, it is less likely to have a large negative loss in Bitcoin. On the other hand, at the upper quantile for the different altcoins, we notice that the 
cross-quantilograms $\hat{p}_{\alpha}(k)$ for $\alpha_{1}<0.5$ are mostly negative and significant, implying that when an altcoin's return is high, there is increased likelihood of having a large negative loss in Bitcoin, while the cross-quantilograms $\hat{p}_{\alpha}(k)$ for $\alpha_{1}>0.5$ are mostly positive, suggesting that when an altcoin's return is high, there is increased likelihood of having a large positive gain in Bitcoin. Exceptions to the above conclusions include Ether and Monero (see Figures 9 and 12, respectively) for which all the sample cross-correlograms illustrate both positive and negative values, while the results are less significant.

Another interesting result that has been found to hold irrespective of the altcoin under consideration is the fact that the cross-quantilogram for $\alpha_{1}=0.5$ is mostly insignificant for both the low and high quantiles of the returns of any altcoin considered and therefore the returns of altcoins are not helpful in predicting whether Bitcoin's price return is located below or above its median. Again, this finding is consistent with the GCQ test results.

Consequently, the results in Figures 8 through 14 illustrate that, although altcoins cannot predict Bitcoin's return under normal market conditions, they are helpful in predicting Bitcoin's extreme price movements. Specifically, we found positive predictability from altcoins to Bitcoin price returns when cryptocurrencies are in either bear or bull market. However, during bull market the altcoins considered reveal negative predictability on Bitcoin's price returns, provided that the price returns of Bitcoin are high. Moreover, during bear market the altcoins considered also reveal negative predictability on Bitcoin returns, provided that the price returns of Bitcoin are relatively low. The above conclusions hold for all the altcoins considered in this study except for Ether and Monero.

\section{Insert Figures 8 through 14 about here}

Our findings therefore indicate that the price returns of Bitcoin and altcoins move in the same direction when they both are in bear or bull market but move in opposite directions when Bitcoin price returns are high and altcoin returns are low or when Bitcoin price returns are low and altcoin returns are high. Furthermore, it has been shown that not only can Bitcoin predict altcoins' extreme price movements but also altcoins can predict Bitcoin's extreme price movements. Our results thus contribute to the growing literature on interdependencies within cryptocurrency markets and illustrate a more complete relationship between Bitcoin and several altcoins as well as how the

relationship changes across time, with detailed features illustrated in the sample cross-quantilograms which depend on the different lags and on each quantile.

\subsubsection{Directional predictability tests between Bitcoin and mainstream assets}

Apart from investigating the predictability between Bitcoin and altcoins, it is also interesting to see whether Bitcoin's price return can predict the price movements of commodity or stock returns. Consequently, we also estimated the cross-quantilograms $\hat{p}_{\alpha}(k)$ for the predictability from Bitcoin 
to gold, oil, and S\&P500 (Figures 15 through 17, respectively), given that Bitcoin's price return is at the extreme quantiles (i.e., 0.1 quantile and 0.9 quantile).

Firstly, we examined the predictability from Bitcoin to gold (Figure 15). The findings demonstrate that when Bitcoin's returns are at the low quantile of $\alpha_{2}=0.1$, and thus Bitcoin is in bear market, the cross-quantilograms $\hat{p}_{\alpha}(k)$ for $\alpha_{1} \leq 0.3$ are negative and significant at most lags providing evidence of negative directional predictability from Bitcoin to gold when both Bitcoin and gold are in bear markets. This result is in accordance with the study of Bouri et al. [2018] who also found evidence that the returns of gold are negatively affected by the volatility of Bitcoin in bear markets. On the other hand, the cross-quantilograms $\hat{p}_{\alpha}(k)$ for $\alpha_{1} \geq 0.7$ are positive and significant on the first 30 days. When the market of Bitcoin is at the high quantile of $\alpha_{2}=0.9$, the crossquantilograms $\hat{p}_{\alpha}(k)$ for $\alpha_{1} \leq 0.3$ are positive and significant, while the cross-quantilograms $\hat{p}_{\alpha}(k)$ for $\alpha_{1} \geq 0.7$ are negative and significant, with the latter finding suggesting negative predictability from Bitcoin to gold when both Bitcoin and gold are in bull market.

Secondly, we investigated the predictability of the oil returns based on Bitcoin's price movements (Figure 16). Similar to the test results for the directional predictability from Bitcoin to gold, the results show that when Bitcoin's returns are at the low quantile, the cross-quantilograms $\hat{p}_{\alpha}(k)$ for $\alpha_{1}$ $\leq 0.3$ are negative and significant at most lags, whereas the cross-quantilogram $\hat{p}_{\alpha}(k)$ for $\alpha_{1}=0.95$ is positive and significant. When Bitcoin's returns are at the high quantile of $\alpha_{2}=0.9$, the crossquantilograms $\hat{p}_{\alpha}(k)$ for $\alpha_{1} \leq 0.3$ are mostly positive and significant, while the cross-quantilogram $\hat{p}_{\alpha}(k)$ for $\alpha_{1}=0.95$ is negative and significant. These results therefore provide evidence of negative predictability from Bitcoin to oil when both Bitcoin and oil are in either bear or bull markets and our results are consistent with those in Bouri et al. [2018].

Thirdly, we assessed the predictability from Bitcoin to S\&P500 (Figure 17). According to the results, when Bitcoin's returns are at the low quantile of $\alpha_{2}=0.1$, the cross-quantilograms $\hat{p}_{\alpha}(k)$ for $\alpha_{1}=0.05$ and 0.1 are positive and significant in the first thirty days, suggesting positive directional predictability, similar to Bouri et al. [2018], and that when Bitcoin's price changes are negative and of very large magnitude, it is very likely that S\&P500 will also exhibit very large negative price changes in the first thirty days, whereas the cross-quantilograms $\hat{p}_{\alpha}(k)$ for $\alpha_{1} \geq 0.7$ are mostly negative but less significant. When Bitcoin's returns are at the upper quantile of $\alpha_{2}=0.9$, the cross-quantilograms $\hat{p}_{\alpha}(k)$ for $\alpha_{1}=0.2$ and 0.3 are mostly positive and significant. Interestingly, the cross-quantilogram $\hat{p}_{\alpha}(k)$ for $\alpha_{1}=0.5$ is also mostly positive and significant, especially during the second month (specifically between 35 and 55 lags), whereas for $\alpha_{1}=0.90$ and 0.95 the results are mixed and mostly insignificant. These results suggest that when Bitcoin is in bull market, it cannot help predict S\&P500's movement when S\&P500 is also in bull market but it can predict S\&P500 at its median as well as when S\&P500 is in bear market.

\section{Insert Figures 15 through 17 about here}


Finally, we estimated the cross-quantilograms $\hat{p}_{\alpha}(k)$ for the predictability from gold, oil, and S\&P500 to Bitcoin (Figures 18 through 20, respectively). Regarding the predictability from gold to Bitcoin (Figure 18), the results illustrate that when the gold market is at the low quantile of $\alpha_{2}=0.1$, the cross-quantilograms $\hat{p}_{\alpha}(k)$ are all positive and significant. There is therefore positive directional predictability in the low quantiles, indicating that when gold's price changes are negative and of very large magnitude, it is very likely that Bitcoin will also experience very large negative price changes in the forthcoming days. Similar results hold for the cross-quantilograms for $\alpha_{1} \leq$ 0.7 when gold's price returns are at the high quantile of $\alpha_{2}=0.9$. However, when the market of Bitcoin is also at a high quantile $\left(\alpha_{1} \geq 0.8\right)$, the cross-quantilograms $\hat{p}_{\alpha}(k)$ become negative. These results thus show positive predictability from gold to Bitcoin when both gold and Bitcoin are in bear markets but negative predictability when both gold and Bitcoin are in bull markets. This result contradicts the previous argument that gold plays a marginal role in determining Bitcoin prices (e.g., Kristoufek [2015] claimed that gold is not a determinant of Bitcoin prices).

Nevertheless, these findings are quite different from those related to the predictability from oil and S\&P500 to Bitcoin. More specifically, regarding the predictability from oil to Bitcoin (Figure 19), it can be noticed that when the oil market is at the low quantile of $\alpha_{2}=0.1$, the crossquantilograms $\hat{p}_{\alpha}(k)$ are negative and significant for $\alpha_{1} \leq 0.3$ but become positive for $\alpha_{1} \geq 0.7$. In contrast, when the oil market is at the high quantile of $\alpha_{2}=0.9$, the cross-quantilograms $\hat{p}_{\alpha}(k)$ are positive and significant for $\alpha_{1} \leq 0.3$ but become negative for $\alpha_{1} \geq 0.7$. As for the predictability from S\&P500 to Bitcoin (Figure 20), when the S\&P500 is at the low quantile, the cross-quantilograms $\hat{p}_{\alpha}(k)$ are mostly negative but close to zero for $\alpha_{1} \leq 0.2$ but become mostly positive for $\alpha_{1} \geq 0.5$. On the other hand, when the S\&P500 is at the upper quantile, the cross-quantilograms $\hat{p}_{\alpha}(k)$ are mostly negative for $\alpha_{1} \geq 0.2$. We have thus found negative predictability from oil and S\&P500 to Bitcoin when both oil/S\&P500 and Bitcoin are in either bear or bull markets.

The above results provide a more comprehensive overview of the relationship between Bitcoin and mainstream assets and are overall in accordance with the findings in the study of Bouri et al. [2018] but are not consistent with previous studies that have found that cryptocurrency markets are isolated from traditional financial markets. Several previous studies argued that Bitcoin is useful as a diversifier because of its very weak correlation with financial assets (see, e.g., Brière et al. [2015]; Baur et al. [2018]; Ji et al., 2018). However, these studies relied on unconditional correlation analysis and did not account for causal relationships using conditional quantile forecasting. As a result, such studies would not be able to provide advice to investors under different market condition scenarios. Our study fills this gap by providing several new insights, with our findings having important implications in terms of diversification benefits and safe-haven properties.

When analysing the predictability from Bitcoin to gold, our results confirmed the asymmetric nature of mean spillovers found in the study of Bouri et al. [2018] in bear and bull markets. Our results also suggest that Bitcoin is not useful as a diversifier when, e.g., both Bitcoin and gold are 
in bear market or when Bitcoin is in bear market and gold is in bull market due to the significant directional predictability witnessed under these market conditions. Our findings thus alert investors and fund managers on the market conditions under which Bitcoin can provide diversification benefits when combining Bitcoin with gold in a portfolio. Accordingly, Bitcoin cannot provide diversification benefits for oil when both are in bear market. Nevertheless, our analysis shows that Bitcoin is a useful diversifier for oil in the market scenario where Bitcoin is in the bull market and oil is in the bear market as a result of insignificant directional predictability, in which case fund managers may consider including oil together with Bitcoin when forming a portfolio. In addition, the positive directional predictability from Bitcoin to S\&P500 found under the scenario where both are in bear market (Figure 17) suggests it is not beneficial to include stock and Bitcoin in the same portfolio when both are under bear market status.

Following Shahzad et al. [2019], we further explore Bitcoin's weak and strong safe-haven properties. According to the authors, Bitcoin is viewed as a strong safe-haven if there is evidence of predictability from an asset to Bitcoin in the low quantiles of both the asset and Bitcoin's returns, and the sign of this predictability is negative, whereas Bitcoin is considered to be a weak safe-haven if there is no evidence of predictability from the asset to Bitcoin in the low quantiles of both the asset and Bitcoin's returns. Consequently, Bitcoin is a weak safe-haven for an asset if no dependence exists between the low quantiles of Bitcoin and the asset (i.e., at the 0.05 quantiles), whereas Bitcoin is a strong safe-haven if extreme negative asset returns are followed by positive Bitcoin returns in the following days, implying negative directional predictability of Bitcoin return from asset returns. In our study, we found no evidence of Bitcoin being a strong safe-haven for S\&P500, especially when Bitcoin's returns are extremely high (see Figure 20). However, there is evidence of the weak safe-haven property for Bitcoin against S\&P500 because no dependence exists between the low quantiles (0.05 quantile). This result is consistent with the study of Shahzad et al. [2019] who also found that Bitcoin can be considered as a weak safe-haven asset for financial indices. We also found that Bitcoin is a strong safe-haven for oil because the negative directional predictability from oil to Bitcoin in the low quantiles (see Figure 19) implies that extreme negative oil returns are followed by positive Bitcoin returns in the next periods. Nevertheless, Bitcoin cannot be considered as either a weak or strong safe-haven for gold because significant positive directional predictability exists between the lower quantiles from gold to Bitcoin (see Figure 18).

These results are therefore of high importance to investors who form portfolios including Bitcoin and traditional assets. As Bitcoin has shown signs of some integration with the asset classes considered in this study, investors and fund managers must be cautious when combining Bitcoin with mainstream assets. Market participants should account for the above relationships under the different market conditions when taking positions in bearish and bullish markets.

\section{Insert Figures 18 through 20 about here}




\section{Concluding comments}

In this study, we examined causal relationships and the potential of improving conditional quantile forecasting between Bitcoin and seven altcoins, namely Ripple, Ether, Stellar, Litecoin, Monero, Dash, and NEM, as well as between Bitcoin and gold, oil, and S\&P500, by employing the Granger-causality in distribution and Granger-causality in quantile tests. It was shown that under normal market conditions the predictability of Bitcoin's price returns conditional on altcoins depends on the altcoin under investigation, whereas none of the commodities or stock considered can be used to forecast Bitcoin during its usual performance. Furthermore, under normal market conditions Bitcoin's price returns were found to significantly Granger-cause only the price returns of the commodities considered but not the price returns of S\&P500 or the price returns of any altcoin. Nevertheless, during extreme market conditions, not only can an enhanced quantile forecast of the altcoins' price returns be produced by conditioning on Bitcoin but also an enhanced quantile forecast of Bitcoin's price returns can be derived by conditioning on an altcoin. This result was even more noticeable during bullish markets. Similar results were also found for the pair-wise causal relationships between Bitcoin and commodities or stock during both superior and poor performance. It was thus shown that it is possible to predict both extreme positive and negative price movements in altcoin, commodity, and stock returns based on Bitcoin as well as to forecast extreme positive and negative movements in Bitcoin's price returns based on the altcoins, commodities, and stock considered.

We also employed the cross-quantilogram which provides a more complete picture on the relationship between two variables in order to detect the direction, duration, and magnitude of the relationship between Bitcoin and altcoins or mainstream assets, showing how the relationship changes at different quantiles and different lags, and thus to test whether Bitcoin can predict altcoin or mainstream asset price movements and vice versa. It was illustrated that Bitcoin's price returns are overall helpful in predicting altcoin price movements when Bitcoin is in either bear or bull market. Similarly, we detected overall positive predictability from altcoins to Bitcoin's price returns when altcoins are in either bear or bull market. However, during bull market Bitcoin's price returns were found to reveal negative predictability on altcoins' price returns, provided that the price returns of altcoins are high, whereas during bear market Bitcoin's returns also show negative predictability on altcoins' returns, provided that the price returns of altcoins are relatively low. Accordingly, during bull market altcoins reveal negative predictability on Bitcoin's price returns, provided that the price returns of Bitcoin are high. Moreover, during bear market altcoins also reveal negative predictability on Bitcoin returns, provided that the price returns of Bitcoin are relatively low. The above results hold for all the altcoins considered in this study except for Ether and Monero. Regarding the predictability from Bitcoin to the commodities and stock considered in our study, we found evidence of negative predictability from Bitcoin to the two commodities when both Bitcoin and gold/oil are in either bear or bull market but positive predictability from Bitcoin 
to S\&P500 when both are in bear market as well as when Bitcoin is in bull market and S\&P500 is in bear market. As for the predictability from mainstream assets to Bitcoin, we found positive predictability from gold to Bitcoin when both are in bear market but negative predictability when both are in bull market. We further found negative predictability from oil and S\&P500 to Bitcoin when both oil/S\&P500 and Bitcoin are in either bear or bull market. Finally, we found evidence that Bitcoin is a strong safe-haven for oil and a weak safe-haven for S\&P500, but it cannot be considered as either a weak or strong safe-haven for gold. Our results are thus in contrast with previous studies that argued that Bitcoin is useful as a diversifier and have important implications in terms of diversification benefits and safe-haven properties.

The issue of cryptocurrency, commodity, and asset predictability is of interest to traders and investors. Our research is also of interest to regulators and policy-makers alike. Continued development of research into the areas of both cryptocurrencies and financial blockchain are central to the development of public support and the ring-fencing of substantial anomalies irregularities that are contained within their structures, whether to support illicit behaviour or not. While portfolio managers continue to observe cryptocurrency markets as a central source of diversification within investment funds, our research presents evidence that cryptocurrency fund investment is quite similar in nature to investment in just a few of the largest cryptocurrencies. Each, while presenting evidence of unique pricing behaviour, are found to be widely interconnected and largely susceptible to sectoral price movements as a whole. Such a finding is very important to generate and develop understanding of the transmission of financial market risk and contagion. The substantial growth in both the price and publicity surrounding cryptocurrencies at large has generated a substantial debate as to the regulatory requirements, the inherent dangers that are sourced within their structure, particularly within the growing number of substantial cases of theft, evidence of market manipulation and other types of illegality that have taken place in recent years. Proponents will continue to point to the benefits of a central placement of blockchain in the modern financial ecosystem. Opponents are also rational when demanding further regulatory influence. It is of the utmost importance that governments, policy-makers and regulators amongst other, continue to develop their understanding of these growing products before creating issues that could potentially generate volatility and contagion effects upon unwilling and unsuspecting financial markets. Despite the continued accusations of illegality, irregular pricing dynamics and evidence of bubble-like behaviour, cryptocurrencies still continue to grow in both demand, complexity and stature. They therefore necessitate a similar regulatory response to match.

\section{Bibliography}

Ardia, D., K. Bluteau, and M. Rüede (2018). Regime changes in (bitcoin) GARCH volatility dynamics. Finance Research Letters Available at: https://doi.org/10.1016/j.frl.2018.08.009. 
Baek, C. and M. Elbeck (2015). Bitcoins as an investment or speculative vehicle? a first look. Applied Economics Letters 22(1), 30-34.

Baumöhl, E. and Š. Lyócsa (2017). Directional predictability from stock market sector indices to gold: A cross-quantilogram analysis. Finance Research Letters 23, 152-164.

Baur, D. G. and T. Dimpfl (2018). Asymmetric volatility in cryptocurrencies. Economics Letters 173, 148-151.

Baur, D. G., T. Dimpfl, and K. Kuck (2017). Bitcoin, gold and the us dollar-a replication and extension. Finance Research Letters.

Baur, D. G., K. Hong, and A. D. Lee (2018). Bitcoin: Medium of exchange or speculative assets? Journal of International Financial Markets, Institutions and Money 54, 177-189.

Blau, B. M. (2018). Price dynamics and speculative trading in bitcoin. Research in International Business and Finance 43, 15-21.

Bouri, E., M. Das, R. Gupta, and D. Roubaud (2018). Spillovers between bitcoin and other assets during bear and bull markets. Applied Economics 50(55), 5935-5949.

Bouri, E., R. Gupta, C. K. M. Lau, D. Roubaud, and S. Wang (2018). Bitcoin and global financial stress: A copula-based approach to dependence and causality in the quantiles. The Quarterly Review of Economics and Finance 69, 297-307.

Bouri, E., S. J. H. Shahzad, and D. Roubaud (2019). Co-explosivity in the cryptocurrency market. Finance Research Letters 29, 178-183.

Brière, M., K. Oosterlinck, and A. Szafarz (2015). Virtual currency, tangible return: Portfolio diversification with bitcoin. Journal of Asset Management 16(6), 365-373.

Brock, W. A., W. D. Dechert, B. LeBaron, and J. A. Scheinkmam (1996). A test for independence based on the correlation dimension. Economic Reviews 15, 197-235.

Cheah, E.-T. and J. Fry (2015). Speculative bubbles in bitcoin markets? an empirical investigation into the fundamental value of bitcoin. Economics Letters 130, 32-36.

Cheah, E.-T., T. Mishra, M. Parhi, and Z. Zhang (2018). Long memory interdependency and inefficiency in bitcoin markets. Economics Letters 16\%, 18-25.

Cheung, A., E. Roca, and J.-J. Su (2015). Crypto-currency bubbles: an application of the phillipsshi-yu (2013) methodology on mt. gox bitcoin prices. Applied Economics 47(23), 2348-2358. 
Ciaian, P., M. Rajcaniova, et al. (2018). Virtual relationships: Short-and long-run evidence from bitcoin and altcoin markets. Journal of International Financial Markets, Institutions and Money 52, $173-195$.

Corbet, S. and P. Katsiampa (2018). Asymmetric mean reversion of bitcoin price returns. International Review of Financial Analysis.

Corbet, S., C. Larkin, B. Lucey, A. Meegan, and L. Yarovaya (2018a). Exploring the dynamic relationships between cryptocurrencies and other financial assets. Economics Letters 165(1), $28-34$.

Corbet, S., C. Larkin, B. Lucey, A. Meegan, and L. Yarovaya (2020). Cryptocurrency reaction to fomc announcements: Evidence of heterogeneity based on blockchain stack position. Journal of Financial Stability 46, 100706.

Corbet, S., C. J. Larkin, B. M. Lucey, A. Meegan, and L. Yarovaya (2018b). The volatility generating effects of macroeconomic news on cryptocurrency returns. Available at SSRN: https://ssrn.com/abstract=3073727.

Corbet, S., B. Lucey, and L. Yarovaya (2018). Datestamping the bitcoin and ethereum bubbles. Finance Research Letters 26, 81-88.

Corbet, S., B. M. Lucey, A. Urquhart, and L. Yarovaya (2018). Cryptocurrencies as a financial asset: A systematic analysis. International Review of Financial Analysis Available at: https://www.sciencedirect.com/science/article/pii/S1057521918305271.

Daniel, K. and D. Hirshleifer (2015). Overconfident investors, predictable returns, and excessive trading. Journal of Economic Perspectives 29(4), 61-88.

Dastgir, S., E. Demir, G. Downing, G. Gozgor, and C. K. M. Lau (2019). The causal relationship between bitcoin attention and bitcoin returns: Evidence from the copula-based granger causality test. Finance Research Letters 28, 160-164.

Diebold, F. X. and K. Yilmaz (2009). Measuring financial asset return and volatility spillovers, with application to global equity markets. The Economic Journal 119(534), 158-171.

Diebold, F. X. and K. Yilmaz (2012). Better to give than to receive: Predictive directional measurement of volatility spillovers. International Journal of Forecasting 28(1), 57-66.

Diebold, F. X. and K. Yllmaz (2014). On the network topology of variance decompositions: Measuring the connectedness of financial firms. Journal of Econometrics 182(1), 119-134. 
Diks, C. and V. Panchenko (2006). A new statistic and practical guidelines for nonparametric granger causality testing. Journal of Economic Dynamics and Control 30(9-10), 1647-1669.

Dyhrberg, A. H. (2016a). Bitcoin, gold and the dollar-a garch volatility analysis. Finance Research Letters 16, 85-92.

Dyhrberg, A. H. (2016b). Hedging capabilities of bitcoin. is it the virtual gold? Finance Research Letters 16, 139-144.

Engle, R. F. and S. Manganelli (2004). Caviar: Conditional autoregressive value at risk by regression quantiles. Journal of Business 83 Economic Statistics 22(4), 367-381.

Fry, J. (2018). Booms, busts and heavy-tails: The story of bitcoin and cryptocurrency markets? Economics Letters 17, 225-229.

Granger, C. W. (1969). Investigating causal relations by econometric models and cross-spectral methods. Econometrica: journal of the Econometric Society, 424-438.

Griffins, J. and A. Shams (2018). Is bitcoin really un-tethered. Available at SSRN 13 June 2018.

Han, H., O. Linton, T. Oka, and Y.-J. Whang (2016). The cross-quantilogram: measuring quantile dependence and testing directional predictability between time series. Journal of Econometrics 193(1), 251-270.

Hill, J. B. (2007). Efficient tests of long-run causation in trivariate var processes with a rolling window study of the money-income relationship. Journal of Applied Econometrics 22(4), 747765 .

Hirshleifer, D. (2001). Investor psychology and asset pricing. The Journal of Finance 56(4), 15331597.

Hong, Y. and H. Li (2004). Nonparametric specification testing for continuous-time models with applications to term structure of interest rates. The Review of Financial Studies 18(1), 37-84.

Jiang, Y., H. Nie, and W. Ruan (2017). Time-varying long-term memory in bitcoin market. Finance Research Letters.

Jiang, Y., H. Nie, and W. Ruan (2018). Time-varying long-term memory in bitcoin market. Finance Research Letters 25, 280-284.

Katsiampa, P. (2017). Volatility estimation for bitcoin: A comparison of garch models. Economics Letters 158, 3-6. 
Katsiampa, P. (2018a). An empirical investigation of volatility dynamics in the cryptocurrency market. Available at: http://dx.doi.org/10.2139/ssrn.3202317.

Katsiampa, P. (2018b). Volatility co-movement between bitcoin and ether. Finance Research Letters Available at: https://doi.org/10.1016/j.frl.2018.10.005.

Katsiampa, P., S. Corbet, and B. Lucey (2019a). High frequency volatility co-movements in cryptocurrency markets. Journal of International Financial Markets, Institutions and Money 62, $35-52$.

Katsiampa, P., S. Corbet, and B. Lucey (2019b). Volatility spillover effects in leading cryptocurrencies: A BEKK-MGARCH analysis. Finance Research Letters 29, 68-74.

Koenker, R. and G. Bassett Jr (1978). Regression quantiles. Econometrica: journal of the Econometric Society, 33-50.

Kristoufek, L. (2015). What are the main drivers of the bitcoin price? evidence from wavelet coherence analysis. PloS one 10(4).

Kumar, S. (2017). On the nonlinear relation between crude oil and gold. Resources Policy 51, 219-224.

Lahiri, S. N. et al. (1999). Theoretical comparisons of block bootstrap methods. The Annals of Statistics 27(1), 386-404.

Lee, T.-H. and W. Yang (2014). Granger-causality in quantiles between financial markets: Using copula approach. International Review of Financial Analysis 33, 70-78.

Linton, O. and Y.-J. Whang (2007). The quantilogram: With an application to evaluating directional predictability. Journal of Econometrics 141(1), 250-282.

Phillip, A., J. Chan, and S. Peiris (2018). A new look at cryptocurrencies. Economics Letters 163, 6-9.

Phillips, P. C., S. Shi, and J. Yu (2015). Testing for multiple bubbles: Historical episodes of exuberance and collapse in the s\&p 500. International Economic Review 56 (4), 1043-1078.

Phillips, P. C., Y. Wu, and J. Yu (2011). Explosive behavior in the 1990s nasdaq: When did exuberance escalate asset values? International Economic Review 52(1), 201-226.

Politis, D. N. and J. P. Romano (1994). The stationary bootstrap. Journal of the American Statistical association 89(428), 1303-1313. 
Shahzad, S. J. H., E. Bouri, D. Roubaud, L. Kristoufek, and B. Lucey (2019). Is bitcoin a better safehaven investment than gold and commodities? International Review of Financial Analysis 63, $322-330$.

Thaler, D. W. F. R. H. (1985). Does the stock market overreact?

Urquhart, A. (2018). What causes the attention of bitcoin? Economics Letters 166, 40-44.

White, H., T.-H. Kim, and S. Manganelli (2015). Var for var: Measuring tail dependence using multivariate regression quantiles. Journal of Econometrics 187(1), 169-188.

Yeh, C.-H. and C.-Y. Yang (2011). Examining the effects of traders' overconfidence on market behavior. In Agent-Based Approaches in Economic and Social Complex Systems VI, pp. 19-31. Springer. 
Figure 1: Cross-quantilogram representing directional predictability from Bitcoin returns to Ripple returns

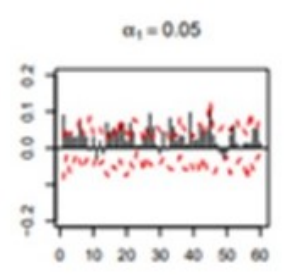

$1 \infty$

$\alpha_{1}=0.3$

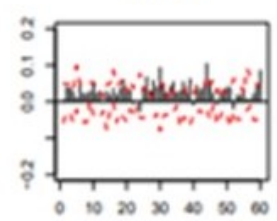

(⿻)

$\alpha_{1}=0.8$

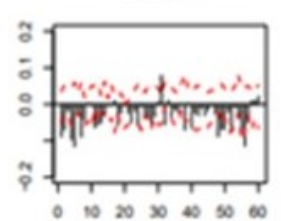

$\ln$

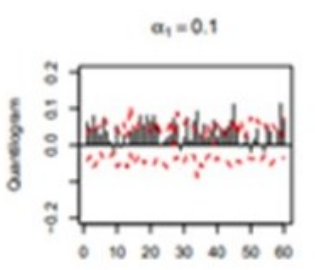

$\ln$

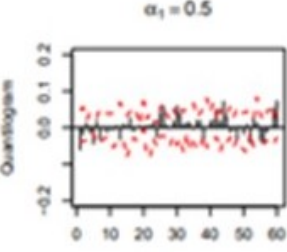

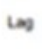

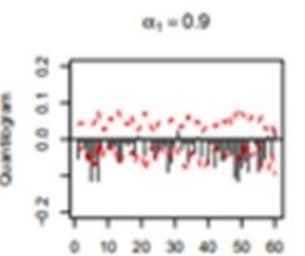

(n)

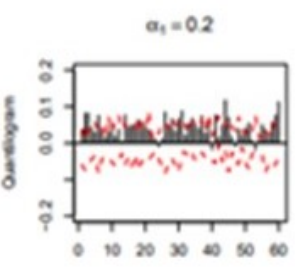

10

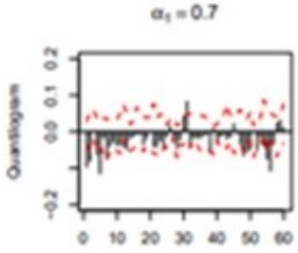

10

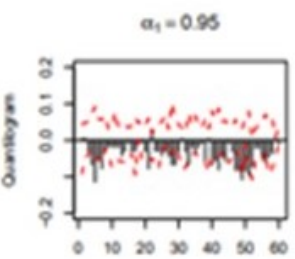

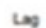

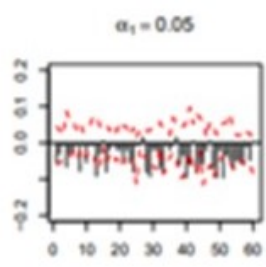

10

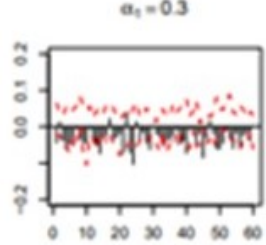

$1 \infty$

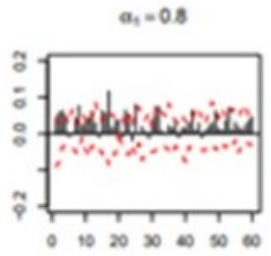

10

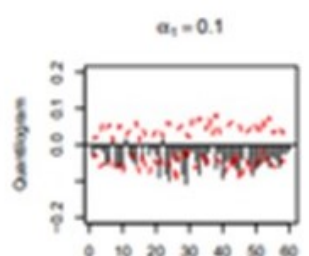

$1 \infty$

$a_{1}=0.5$

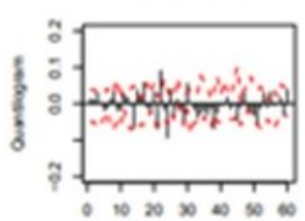

(⿻)

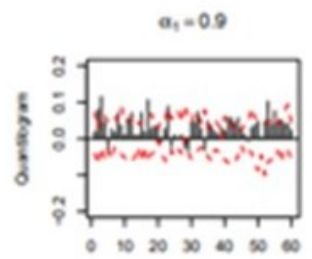

(n)

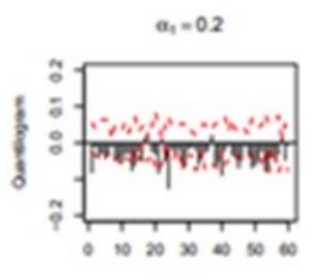

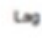

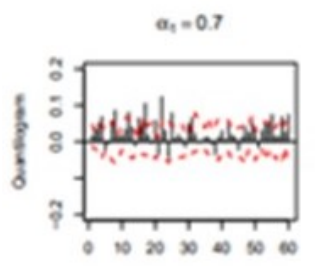

(⿻)

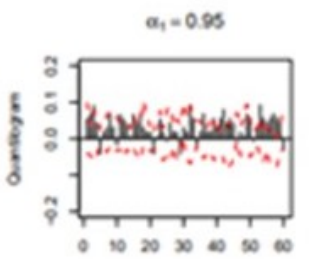

in

a) $\alpha_{2}=0.1$

b) $\alpha_{2}=0.9$

Source: To detect directional predictability from Bitcoin returns to Ripple returns, the sample cross quantilogram $\hat{p}_{\alpha}(k)$ for $\alpha_{2}=0.1$ and $\hat{p}_{\alpha}(k)$ for $\alpha_{2}=0.9$. The bar graphs explain sample cross quantilograms, and the lines are the $95 \%$ bootstrap confidence intervals. 
Figure 2: Cross-quantilogram representing directional predictability from Bitcoin returns to Ether returns

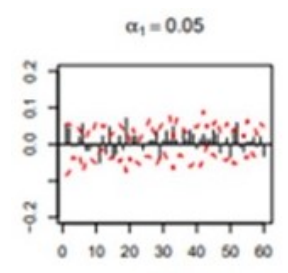

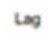

$\alpha_{1}=0.3$

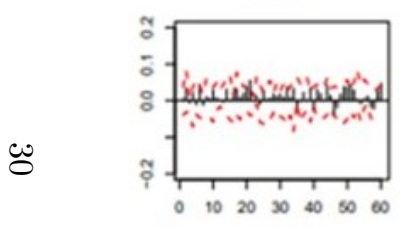

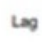

$\alpha_{1}=0.8$

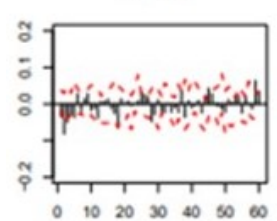

$\operatorname{lng}$

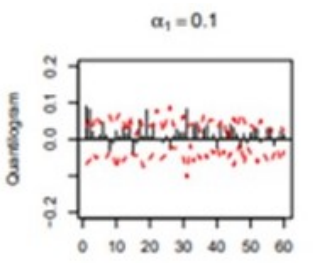

$\stackrel{40}{\infty}$

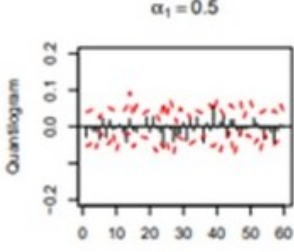

Los

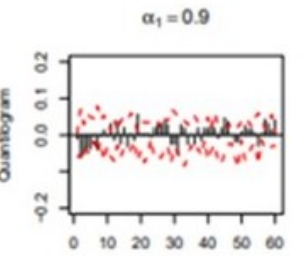

Las

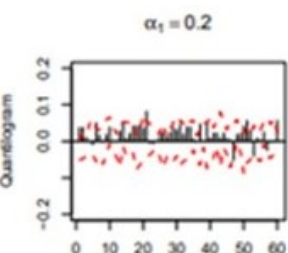

100

$\alpha_{3}=0.7$

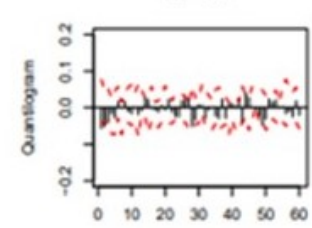

Los

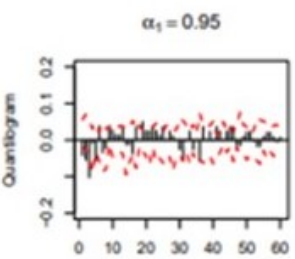

Log

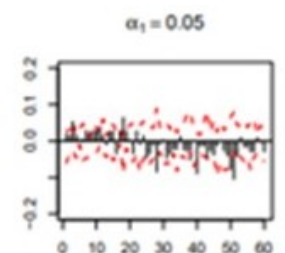

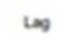

$a_{1}=03$

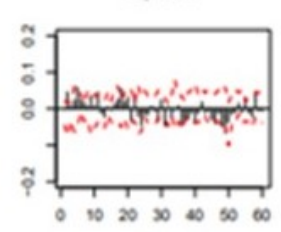

Lis

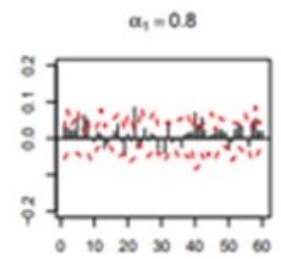

in

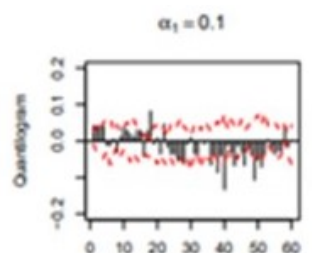

$\rightarrow$

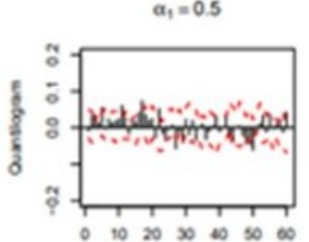

$\infty$

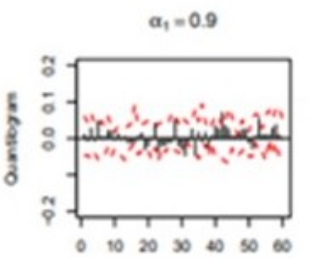

Lis

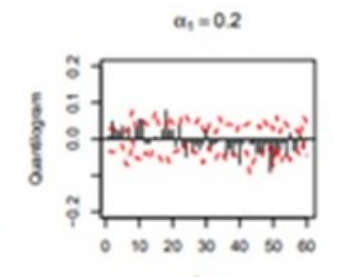

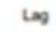

$\alpha_{1}=0.7$

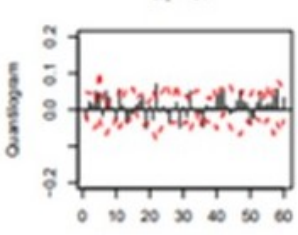

$\rightarrow$

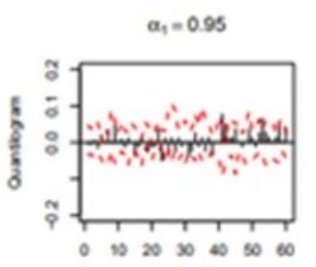

$\infty$

a) $\alpha_{2}=0.1$

b) $\alpha_{2}=0.9$

Source: To detect directional predictability from Bitcoin returns to Ether returns, the sample cross quantilogram $\hat{p}_{\alpha}(k)$ for $\alpha_{2}=0.1$ and $\hat{p}_{\alpha}(k)$ for $\alpha_{2}=0.9$. The bar graphs explain sample cross quantilograms, and the lines are the $95 \%$ bootstrap confidence intervals. 
Figure 3: Cross-quantilogram representing directional predictability from Bitcoin returns to Stellar returns

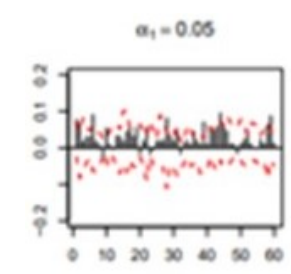

$1 \infty$

$a_{3}=03$

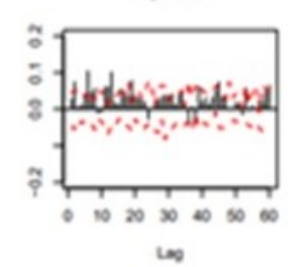

$\alpha_{4}=0.8$

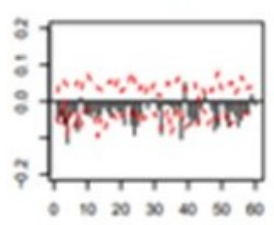

$1 \infty$

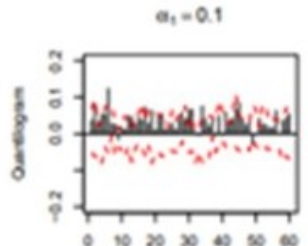

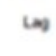

$a_{1}=0.5$
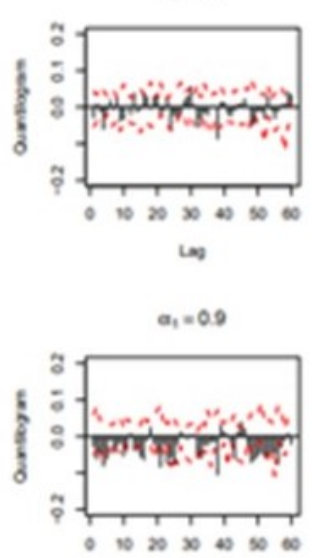

$\ln$

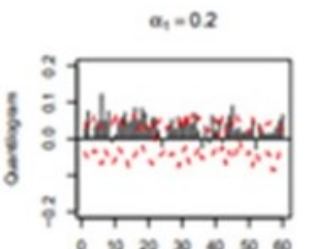

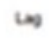

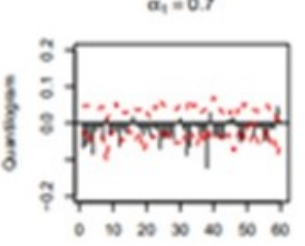

40

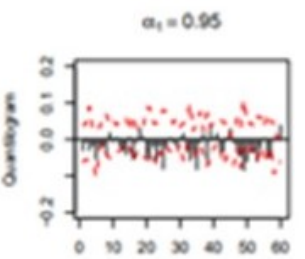

$\infty$

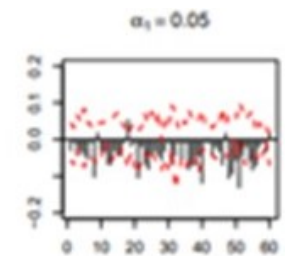

in

$a_{4}=03$

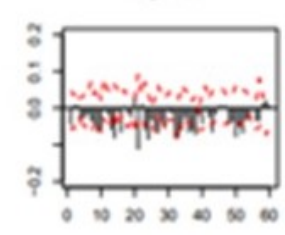

un

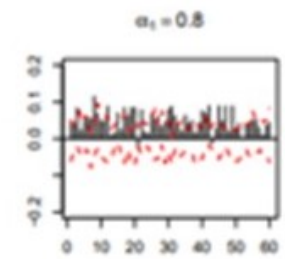

(⿻)

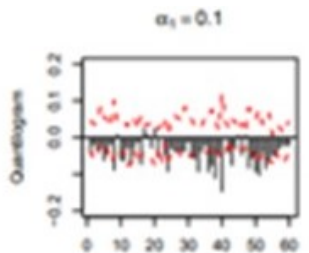

in

$\alpha_{1}=0.5$

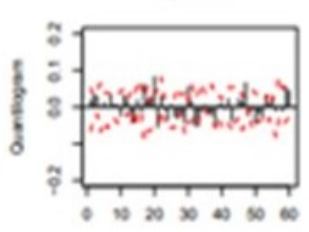

in

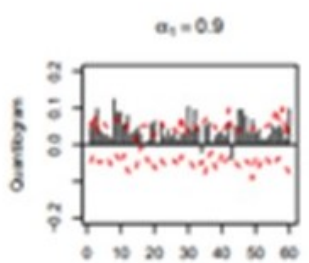

4

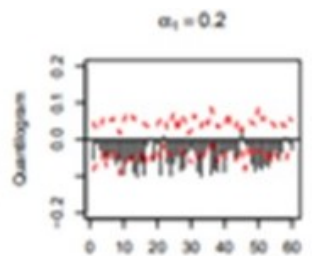

$\infty$

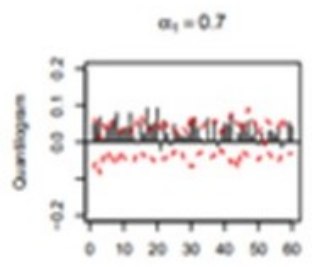

is

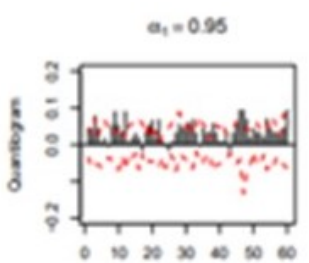

$\infty$

a) $\alpha_{2}=0.1$

b) $\alpha_{2}=0.9$

Source: To detect directional predictability from Bitcoin returns to Stellar returns, the sample cross quantilogram $\hat{p}_{\alpha}(k)$ for $\alpha_{2}=0.1$ and $\hat{p}_{\alpha}(k)$ for $\alpha_{2}=0.9$. The bar graphs explain sample cross quantilograms, and the lines are the $95 \%$ bootstrap confidence intervals. 
Figure 4: Cross-quantilogram representing directional predictability from Bitcoin returns to Litecoin returns

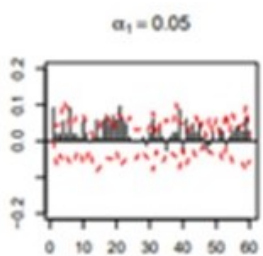

(n)

$a_{1}=0.3$

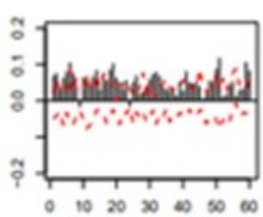

ᄂ

$a_{1}=0.8$

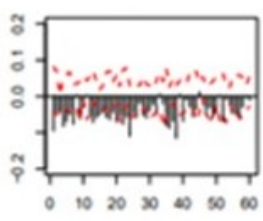

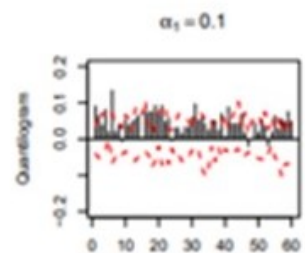

$1 m$

$a_{1}=0.5$

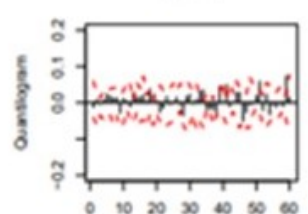

$\ln$

$\alpha_{1}=0.9$

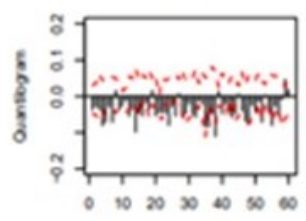

$\alpha_{2}=0$.

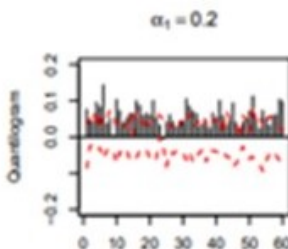

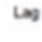

$\alpha_{1}=0.7$

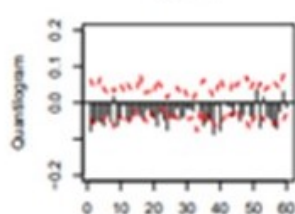

$\infty$

$\alpha_{1}=0.95$

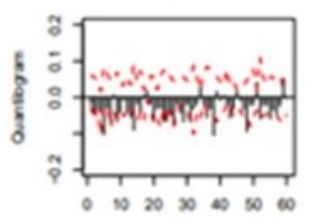

Lis

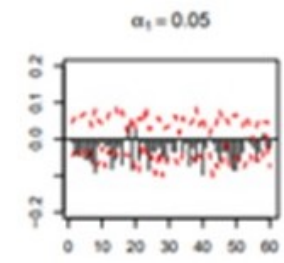

4

$a_{1}=0.3$

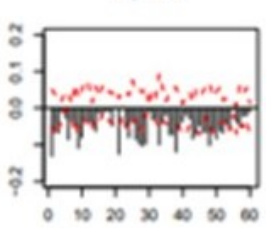

4

$\alpha_{1}=0.8$

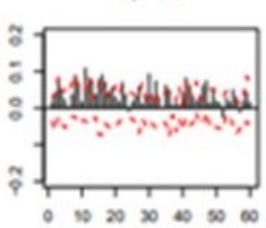

4

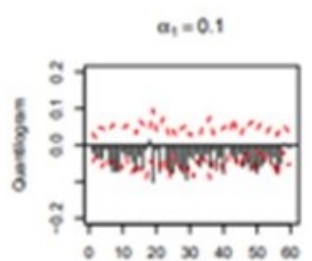

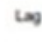

$a_{3}=0.5$

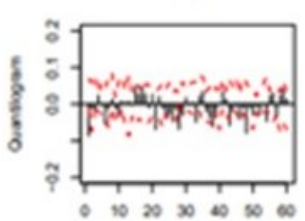

$\omega$

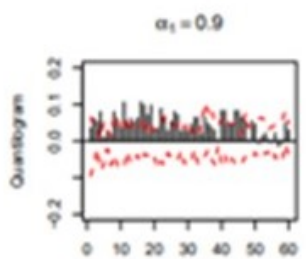

in

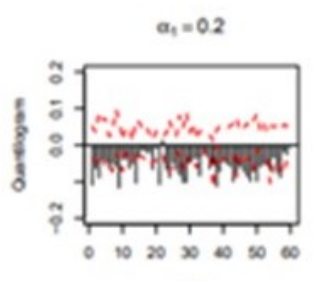

$w$

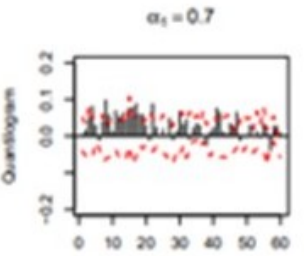

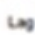

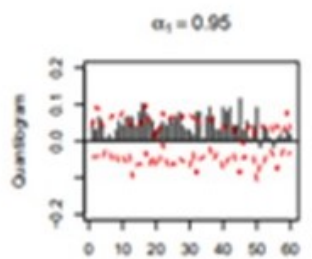

$\omega$

Source: To detect directional predictability from Bitcoin returns to Litecoin returns, the sample cross quantilogram $\hat{p}_{\alpha}(k)$ for $\alpha_{2}=0.1$ and $\hat{p}_{\alpha}(k)$ for $\alpha_{2}=0.9$. The bar graphs explain sample cross quantilograms, and the lines are the $95 \%$ bootstrap confidence intervals. 
Figure 5: Cross-quantilogram representing directional predictability from Bitcoin returns to Monero returns

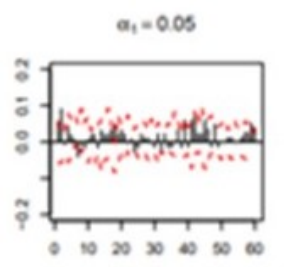

in

$a_{1}=0.3$

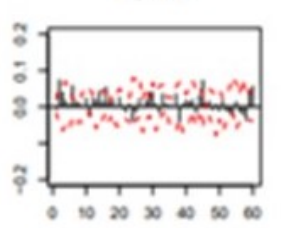

10

$a_{4}=0.8$

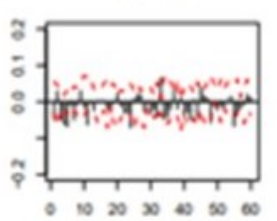

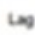

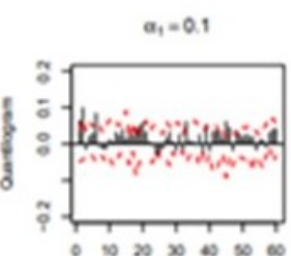

w

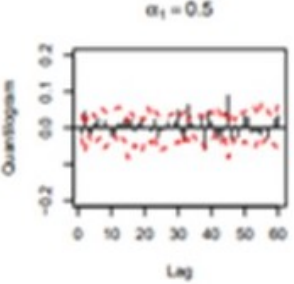

$a_{1}=0$,

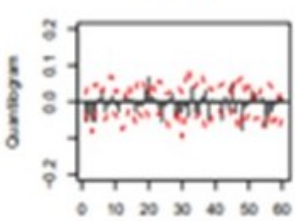

in

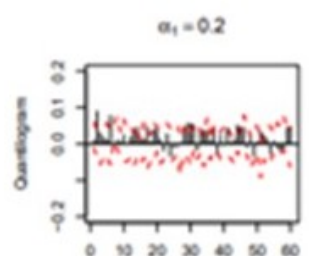

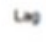

$a_{1}=0.7$
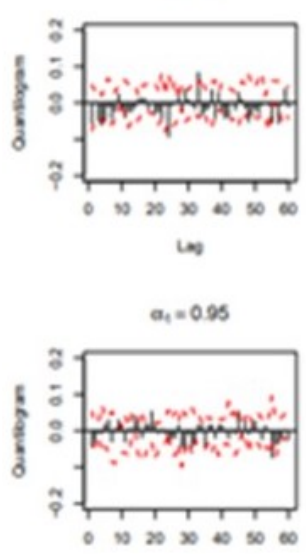

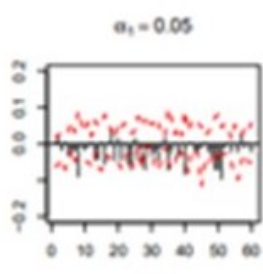

10

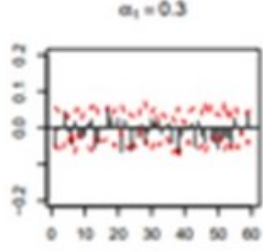

(⿻心㇒

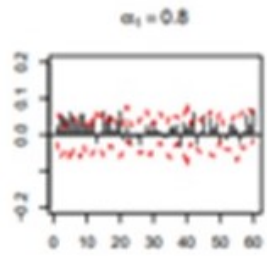

4

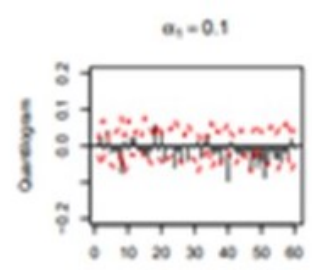

10

$a_{3}=0.5$

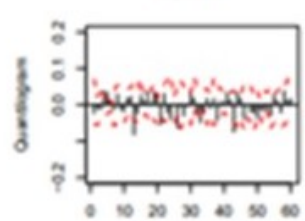

$\infty$

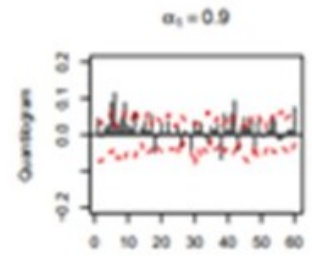

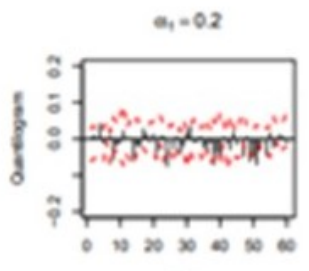

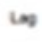

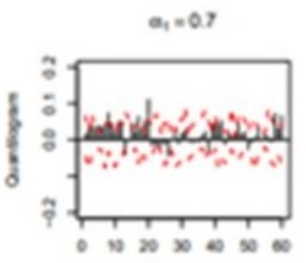

us

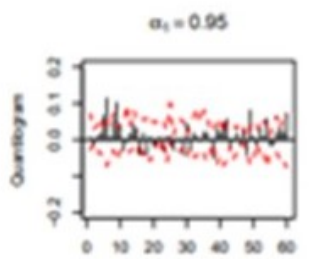

is

a) $\alpha_{2}=0.1$

b) $\alpha_{2}=0.9$

Source: To detect directional predictability from Bitcoin returns to Monero returns, the sample cross quantilogram $\hat{p}_{\alpha}(k)$ for $\alpha_{2}=0.1$ and $\hat{p}_{\alpha}(k)$ for $\alpha_{2}=0.9$. The bar graphs explain sample cross quantilograms, and the lines are the $95 \%$ bootstrap confidence intervals. 
Figure 6: Cross-quantilogram representing directional predictability from Bitcoin returns to Dash returns

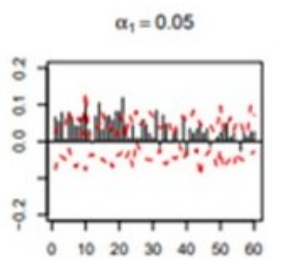

$\operatorname{Ln}$

$\alpha_{1}=0.3$

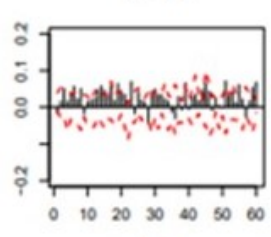

$\infty$

$\alpha_{1}=0.8$

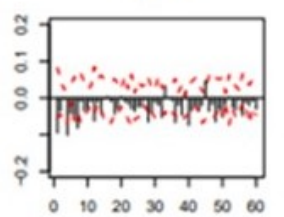

Lo

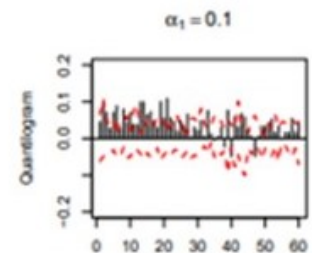

$1 \infty$

$\alpha_{1}=0.5$

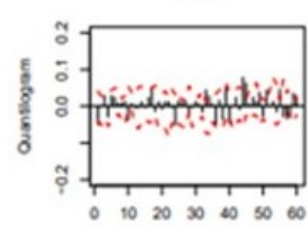

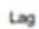

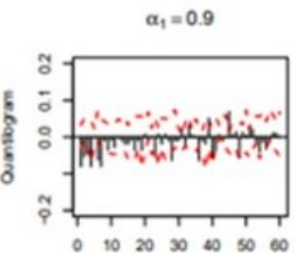

$\infty$

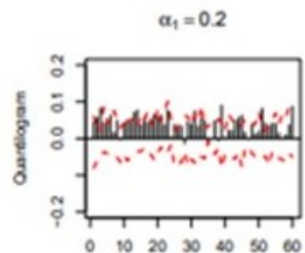

Las
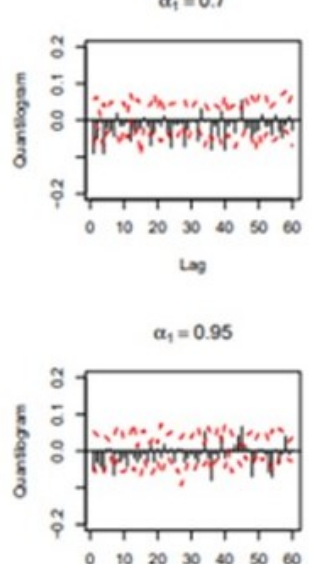

$\ln$

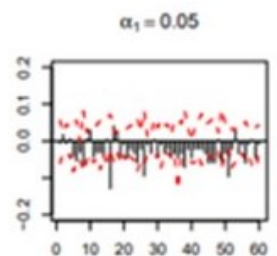

$\operatorname{Ln}$

$\alpha_{1}=0.3$

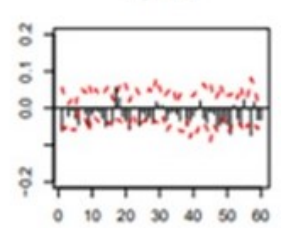

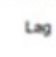

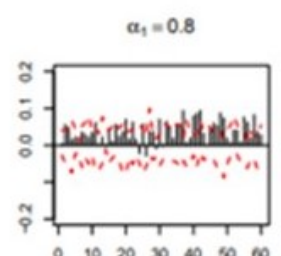

ing

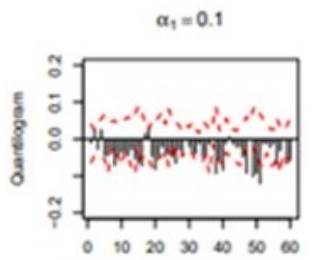

Lag

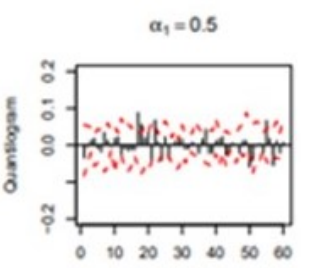

La

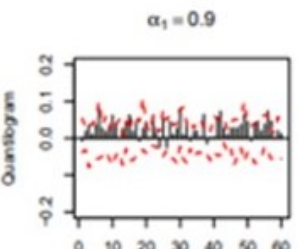

Lag

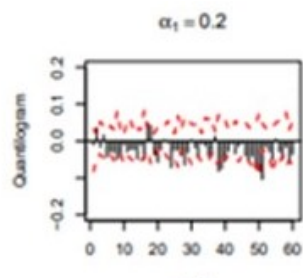

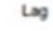

$\alpha_{1}=0.7$

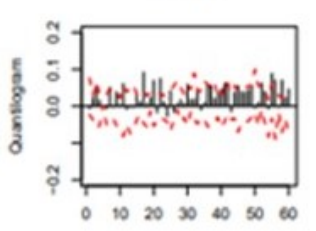

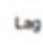

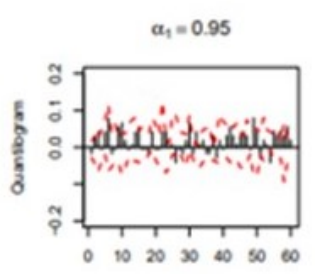

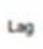

a) $\alpha_{2}=0.1$

b) $\alpha_{2}=0.9$

Source: To detect directional predictability from Bitcoin returns to Dash returns, the sample cross quantilogram $\hat{p}_{\alpha}(k)$ for $\alpha_{2}=0.1$ and $\hat{p}_{\alpha}(k)$ for $\alpha_{2}=0.9$ The bar graphs explain sample cross quantilograms, and the lines are the $95 \%$ bootstrap confidence intervals. 
Figure 7: Cross-quantilogram representing directional predictability from Bitcoin returns to NEM returns

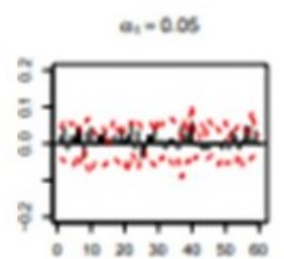

in

$0,-0.3$

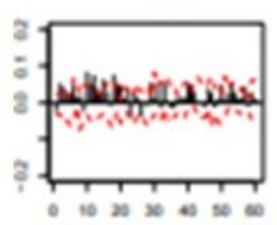

us

$a,=0.8$

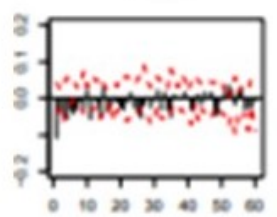

in

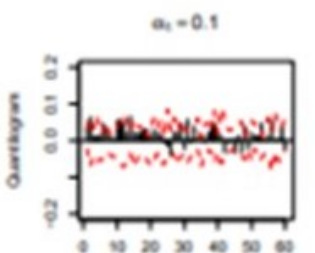

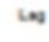

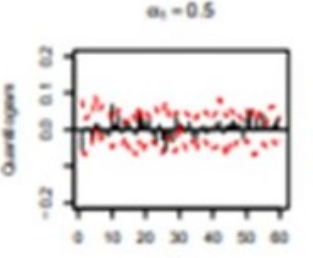

$a,=0.9$

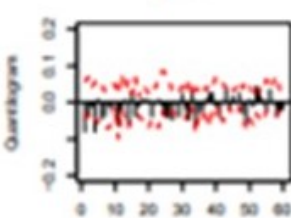

$+\infty$

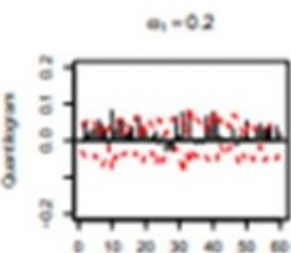

10

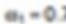

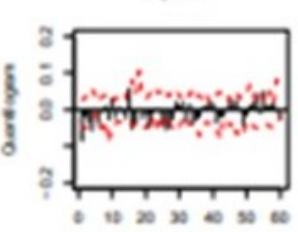

4

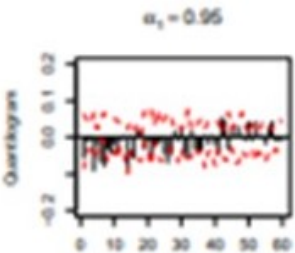

ins

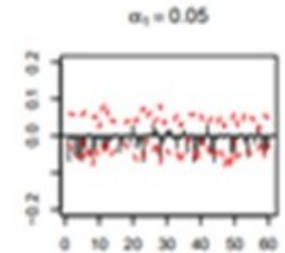

in

a. $=0,3$

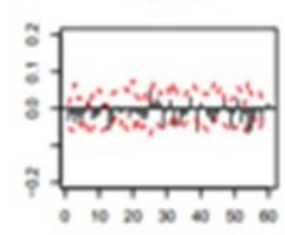

$a_{1}=0.8$

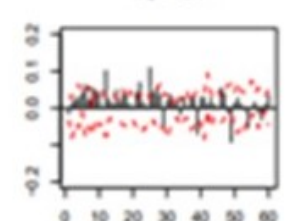

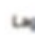

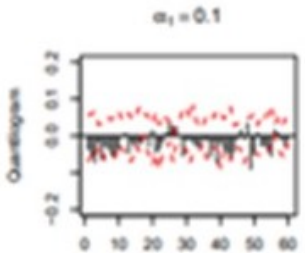

(4)

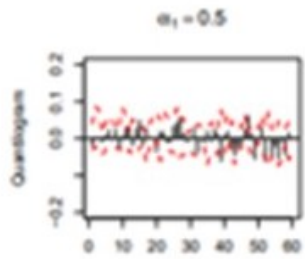

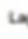

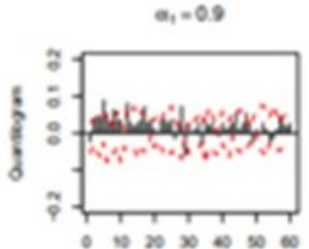

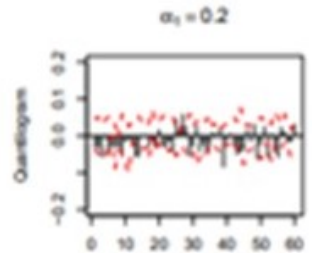

in

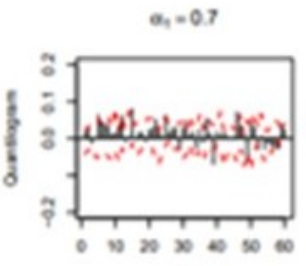

เ

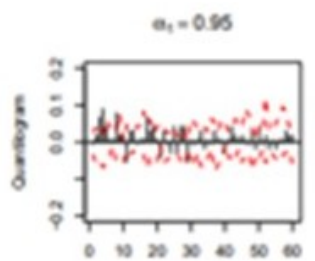

us

a) $\alpha_{2}=0.1$

b) $\alpha_{2}=0.9$

Source: To detect directional predictability from Bitcoin returns to NEM returns, the sample cross quantilogram $\hat{p}_{\alpha}(k)$ for $\alpha_{2}=0.1$ and $\hat{p}_{\alpha}(k)$ for $\alpha_{2}=0.9$ The bar graphs explain sample cross quantilograms, and the lines are the $95 \%$ bootstrap confidence intervals. 
Figure 8: Cross-quantilogram representing directional predictability from Ripple returns to Bitcoin returns

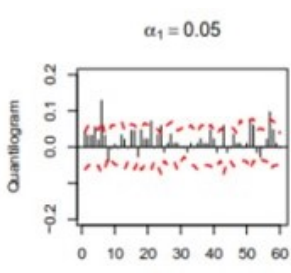

Lag

$\alpha_{1}=0.3$

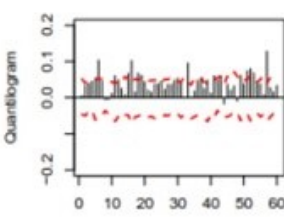

Loo

$\alpha_{1}=0.8$

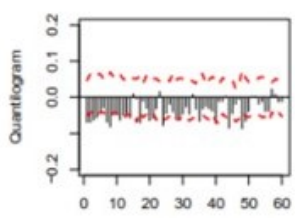

Lag

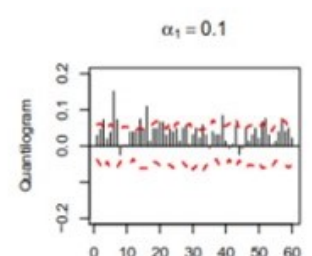

Las

$\alpha_{1}=0.5$

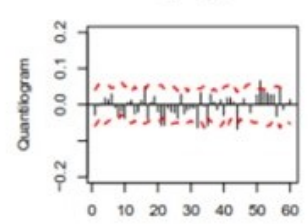

Los

$\alpha_{1}=0.9$

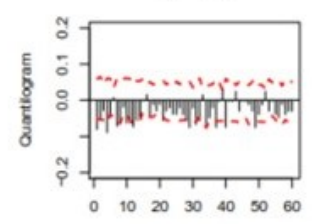

Lag

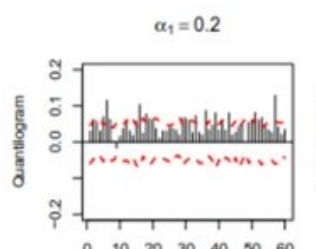

Lag

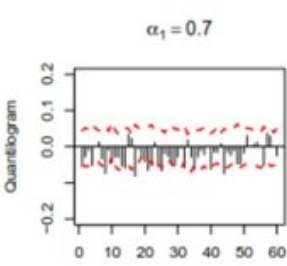

Loo

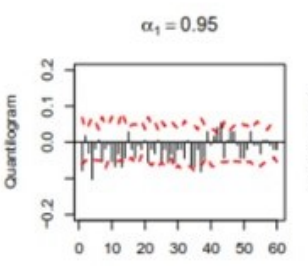

Loo

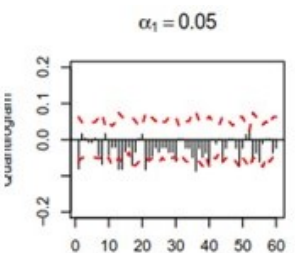

Lag

$\alpha_{1}=0.3$

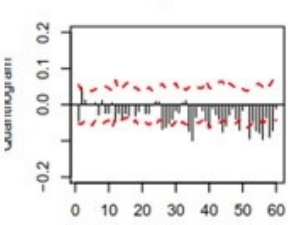

Lag

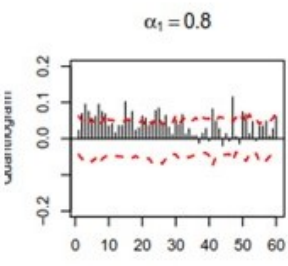

Lag

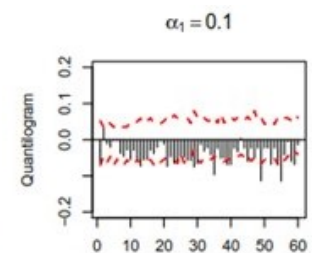

Lag

$\alpha_{1}=0.5$

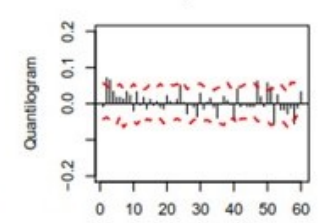

Lag

$\alpha_{1}=0$.

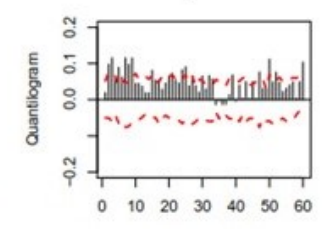

Lag

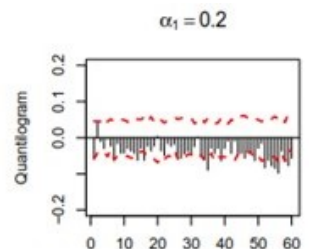

Lag

$\alpha_{1}=0.7$

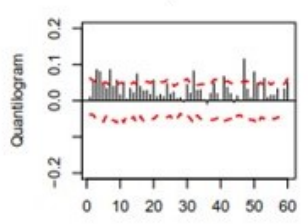

Lag

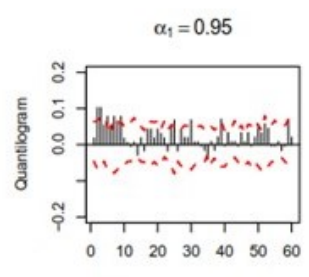

Lag

a) $\alpha_{2}=0.1$

b) $\alpha_{2}=0.9$

Source: To detect directional predictability from Ripple returns to Bitcoin returns, the sample cross quantilogram $\hat{p}_{\alpha}(k)$ for $\alpha_{2}=0.1$ and $\hat{p}_{\alpha}(k)$ for $\alpha_{2}=0.9$ The bar graphs explain sample cross quantilograms, and the lines are the $95 \%$ bootstrap confidence intervals. 
Figure 9: Cross-quantilogram representing directional predictability from Ether returns to Bitcoin returns

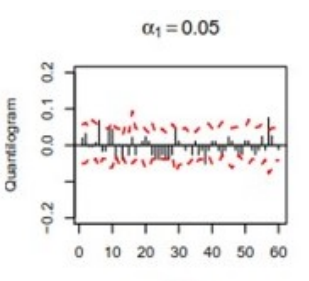

Lag

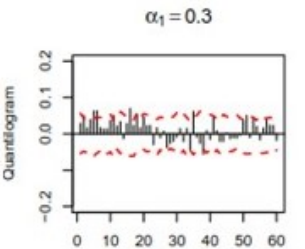

Lag

$\alpha_{1}=0.8$

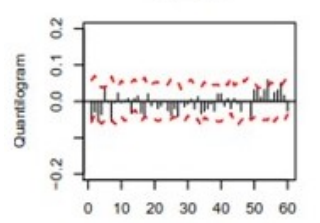

Lag

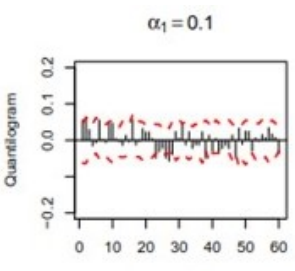

Lag

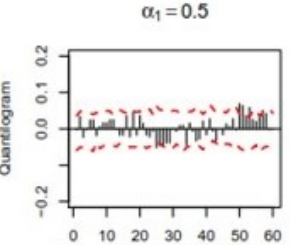

Lag

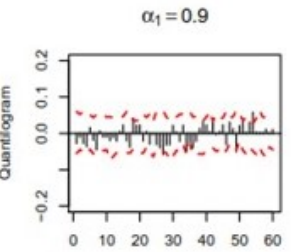

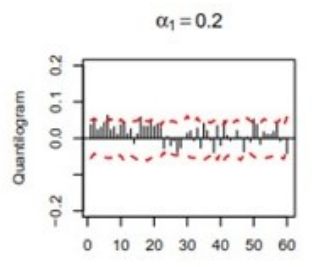

Lag

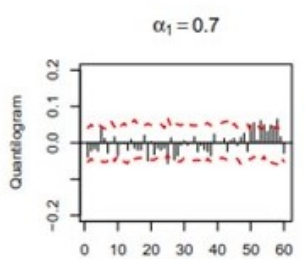

Lag

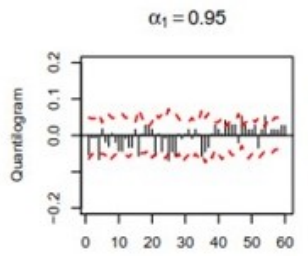

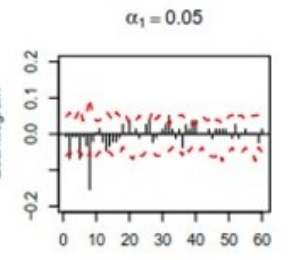

Lag

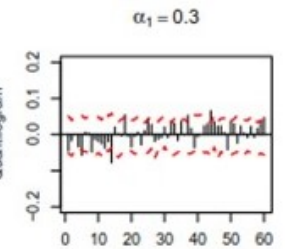

Lag

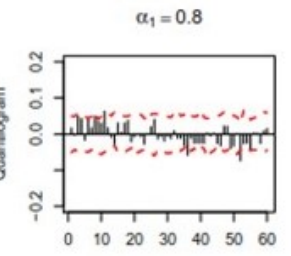

Lag

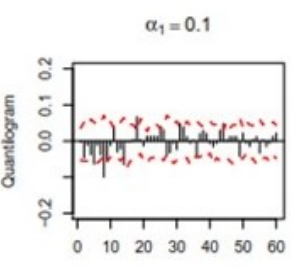

Lag

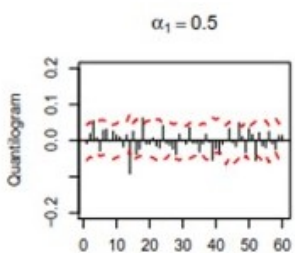

Lag

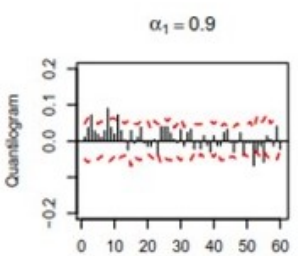

Lag

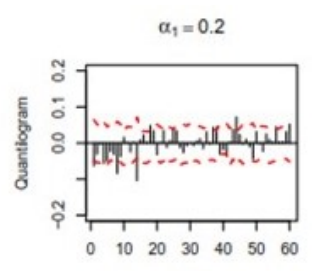

Lag

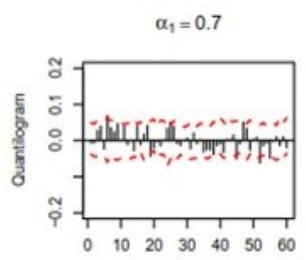

Las

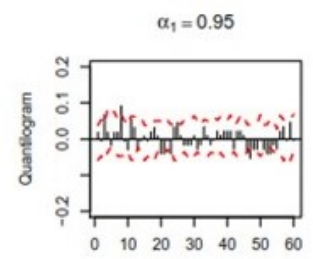

b) $\alpha_{2}=0.9$

Source: To detect directional predictability from Ether returns to Bitcoin returns, the sample cross quantilogram $\hat{p}_{\alpha}(k)$ for $\alpha_{2}=0.1$ and $\hat{p}_{\alpha}(k)$ for $\alpha_{2}=0.9$. The bar graphs explain sample cross quantilograms, and the lines are the $95 \%$ bootstrap confidence intervals. 
Figure 10: Cross-quantilogram representing directional predictability from Stellar returns to Bitcoin returns

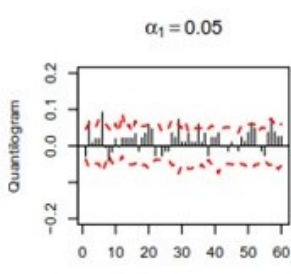

Lag

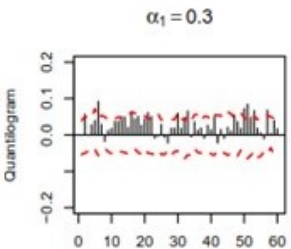

Lag

$\alpha_{1}=0.8$

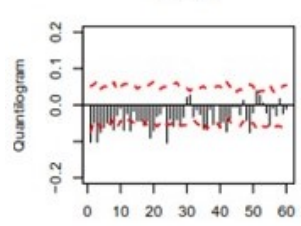

Lag

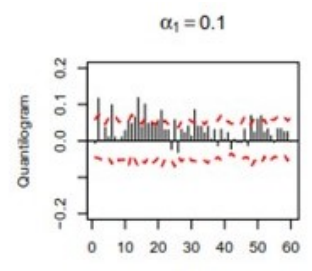

Lag

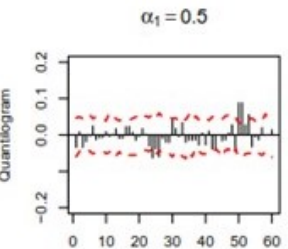

Lag

$\alpha_{1}=0.9$

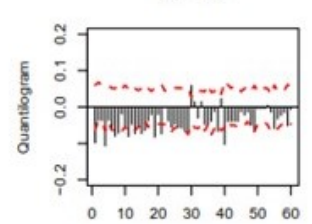

Lag

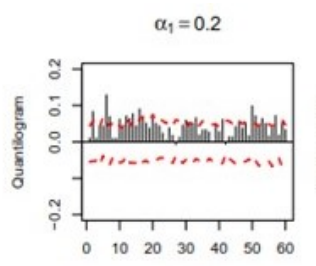

Lag
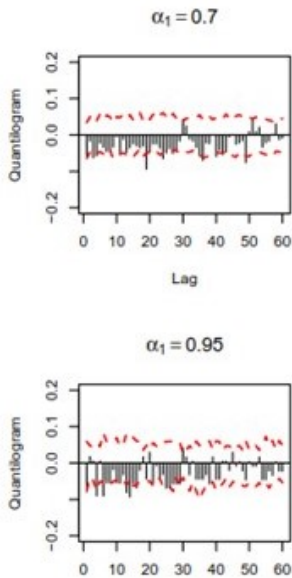

Lag

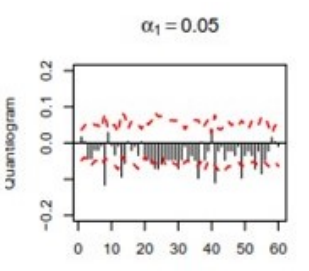

Lag
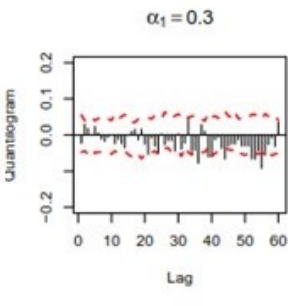

$\alpha_{1}=0.8$

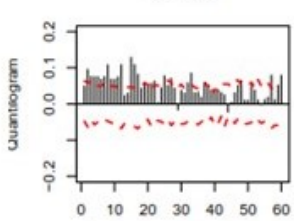

Lag

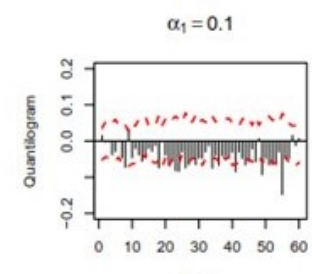

Lag

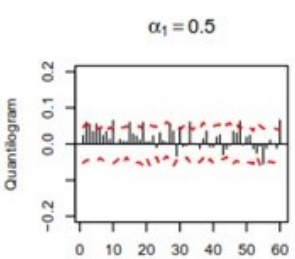

Lag

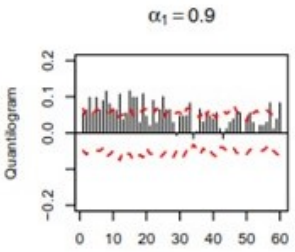

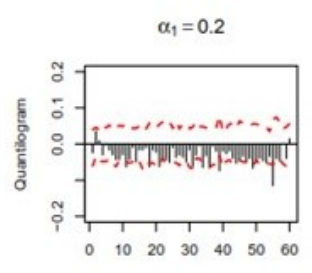

Lag

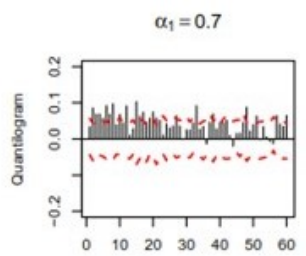

Lag

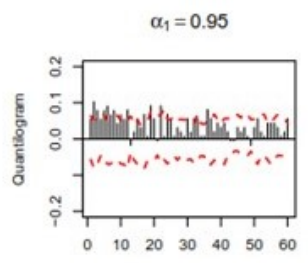

Lag

b) $\alpha_{2}=0.9$

Source: To detect directional predictability from Stellar returns to Bitcoin returns, the sample cross quantilogram $\hat{p}_{\alpha}(k)$ for $\alpha_{2}=0.1$ and $\hat{p}_{\alpha}(k)$ for $\alpha_{2}=0.9$. The bar graphs explain sample cross quantilograms, and the lines are the $95 \%$ bootstrap confidence intervals. 
Figure 11: Cross-quantilogram representing directional predictability from Litecoin returns to Bitcoin returns

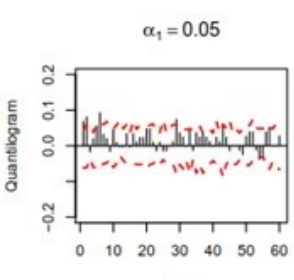

Lag

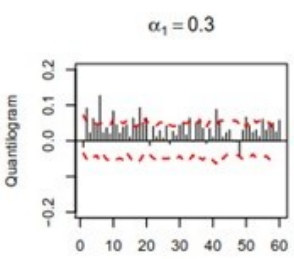

Lag

$\alpha_{1}=0.8$

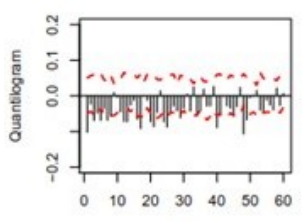

Lag

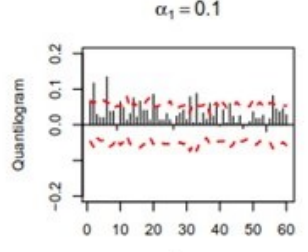

Lag

$\alpha_{1}=0.5$

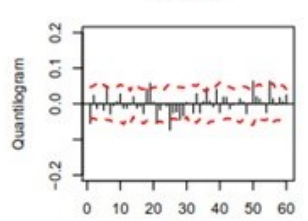

$\alpha_{1}=0.9$

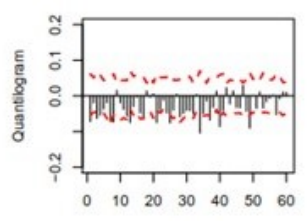

Lag

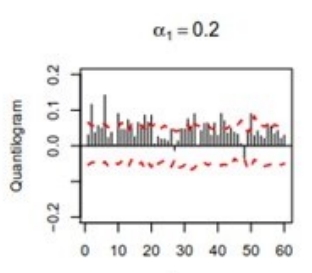

Lag

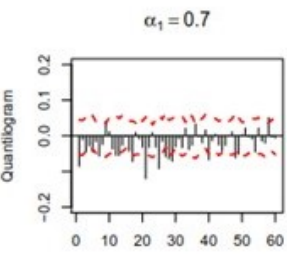

$\alpha_{1}=0.95$

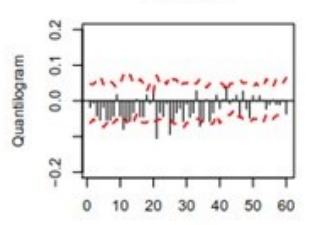

Lag

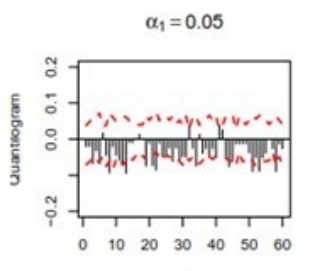

Lag

$\alpha_{1}=0.3$
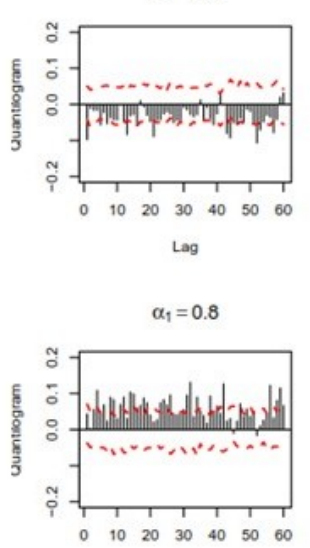

Lag

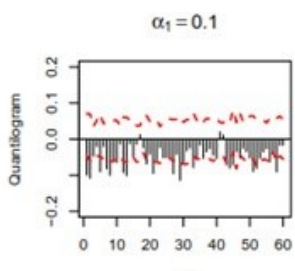

Lag

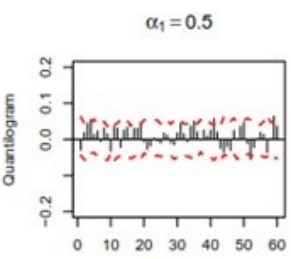

Lag

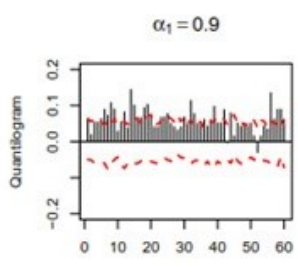

Lag

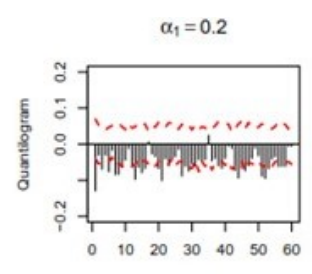

Lag

$\alpha_{1}=0.7$
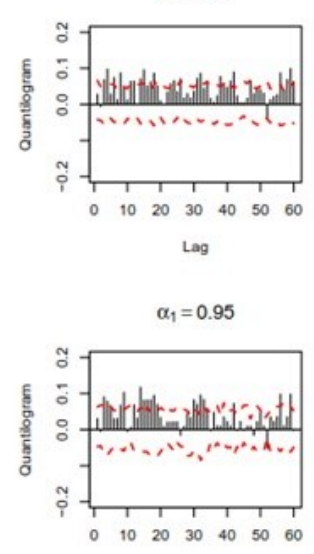

Lag

$$
\text { a) } \alpha_{2}=0.1
$$

b) $\alpha_{2}=0.9$

Source: To detect directional predictability from Litecoin returns to Bitcoin returns, the sample cross quantilogram $\hat{p}_{\alpha}(k)$ for $\alpha_{2}=0.1$ and $\hat{p}_{\alpha}(k)$ for $\alpha_{2}=0.9$ The bar graphs explain sample cross quantilograms, and the lines are the $95 \%$ bootstrap confidence intervals. 
Figure 12: Cross-quantilogram representing directional predictability from Monero returns to Bitcoin returns

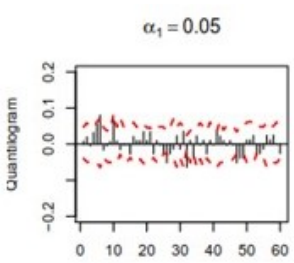

Lag

$\alpha_{1}=0.3$

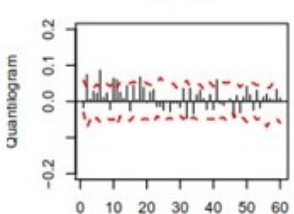

Lag

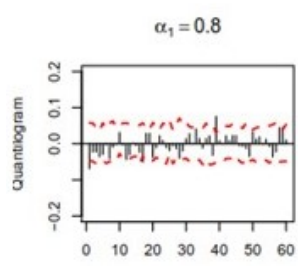

Lag

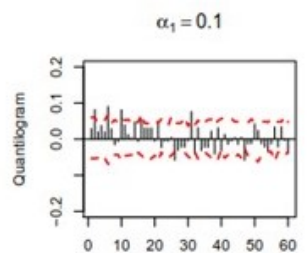

Lag

$\alpha_{1}=0.5$

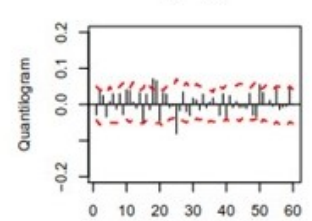

Lag

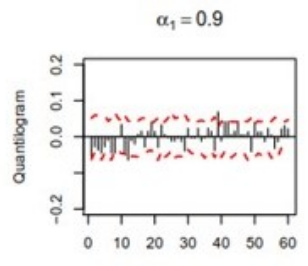

Lag

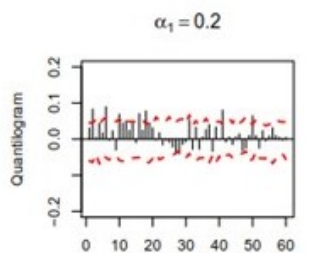

Lag

$\alpha_{1}=0.7$

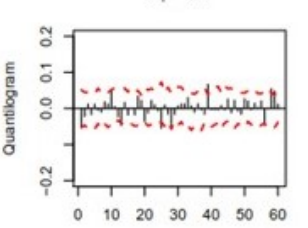

Lag

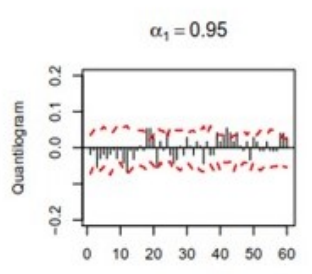

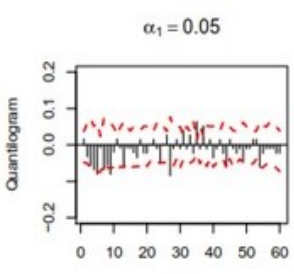

Lag

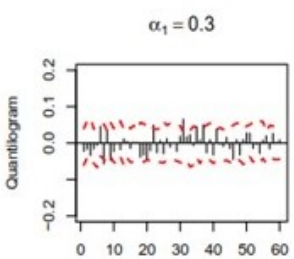

Lag

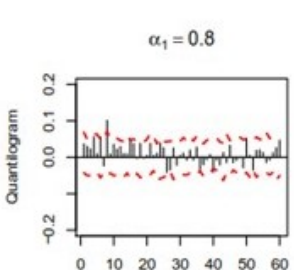

Lag

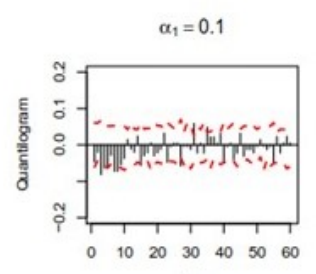

$\operatorname{Lag}$

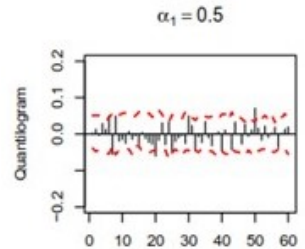

Lag

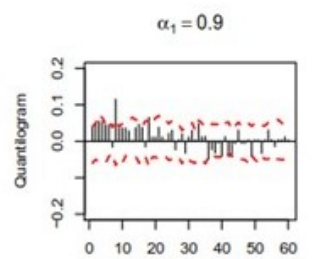

Log

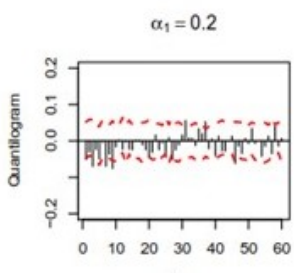

Lag

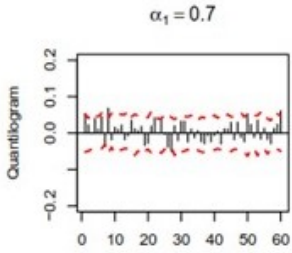

Lag

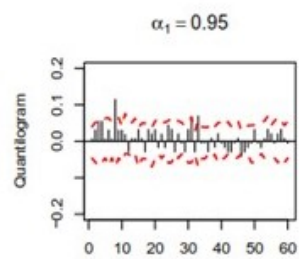

Lag

a) $\alpha_{2}=0.1$

b) $\alpha_{2}=0.9$

Source: To detect directional predictability from Monero returns to Bitcoin returns, the sample cross quantilogram $\hat{p}_{\alpha}(k)$ for $\alpha_{2}=0.1$ and $\hat{p}_{\alpha}(k)$ for $\alpha_{2}=0.9$ The bar graphs explain sample cross quantilograms, and the lines are the $95 \%$ bootstrap confidence intervals. 
Figure 13: Cross-quantilogram representing directional predictability from Dash returns to Bitcoin returns

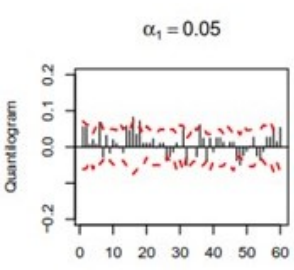

Lag

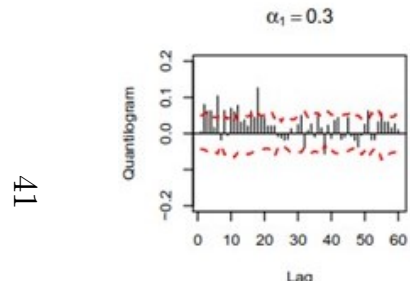

$\alpha_{1}=0.8$

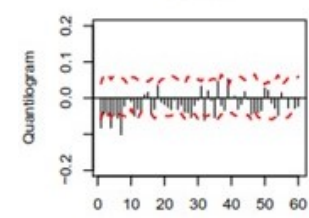

Lag

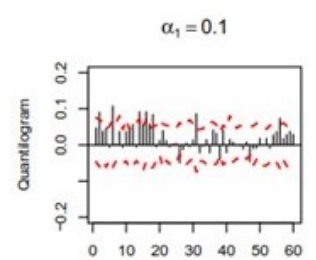

Lag

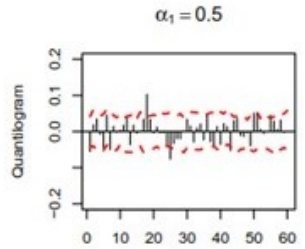

La

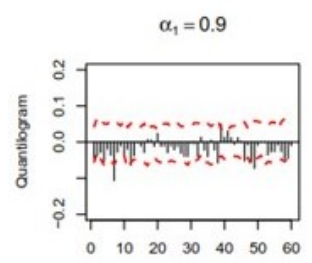

Lag

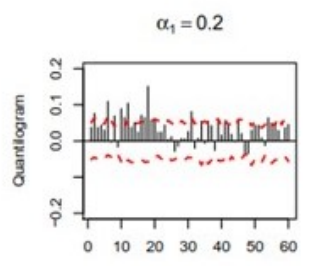

Lag

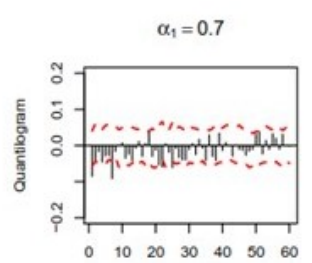

Las

$\alpha_{1}=0.95$

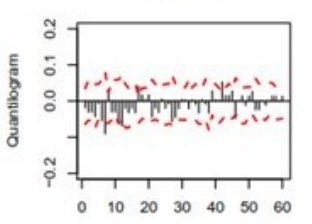

Lag $\alpha_{1}=0.05$

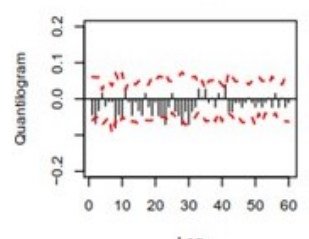

Lag

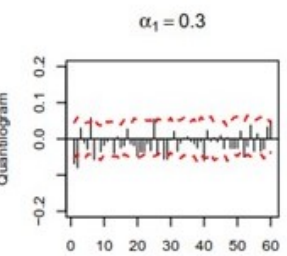

Lag

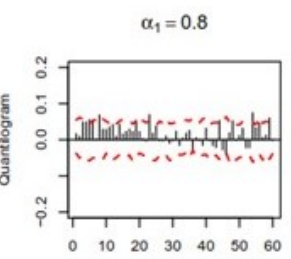

Lag

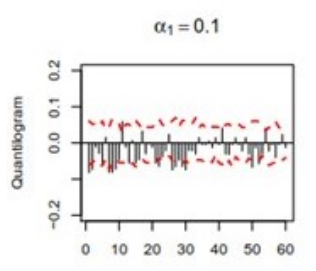

Lag

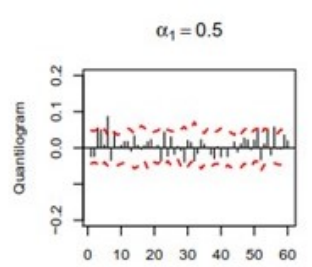

Lag

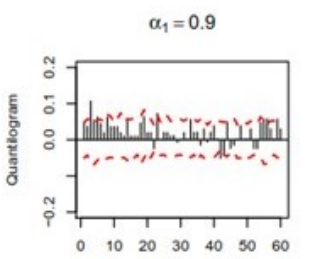

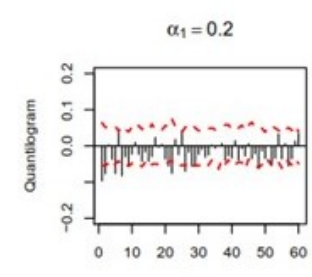

Lag

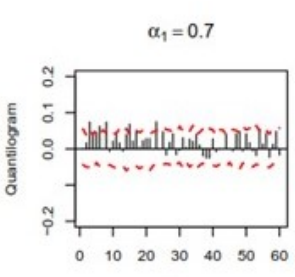

Lag

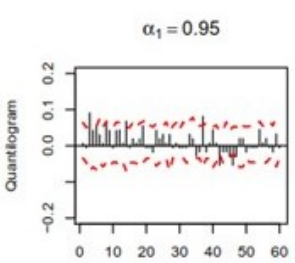

Lag

a) $\alpha_{2}=0.1$

b) $\alpha_{2}=0.9$

Source: To detect directional predictability from Dash returns to Bitcoin returns, the sample cross quantilogram $\hat{p}_{\alpha}(k)$ for $\alpha_{2}=0.1$ and $\hat{p}_{\alpha}(k)$ for $\alpha_{2}=0.9$. The bar graphs explain sample cross quantilograms, and the lines are the $95 \%$ bootstrap confidence intervals. 
Figure 14: Cross-quantilogram representing directional predictability from NEM returns to Bitcoin returns

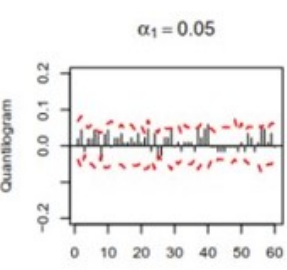

Lag

$\alpha_{1}=0.3$

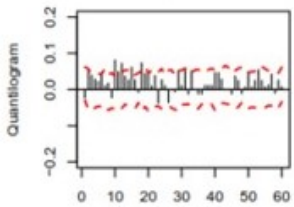

Las

$\alpha_{1}=0.8$

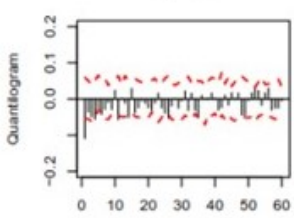

Las

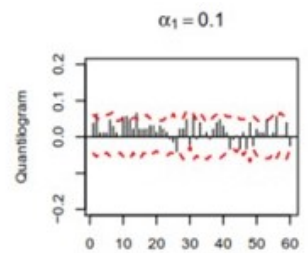

Lag

$\alpha_{1}=0.5$
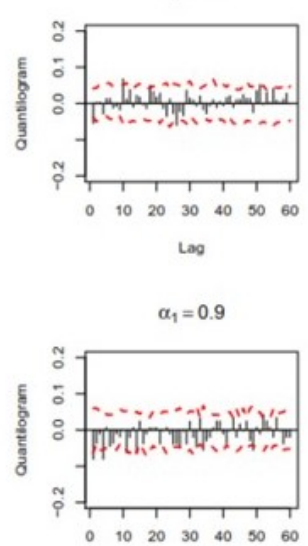

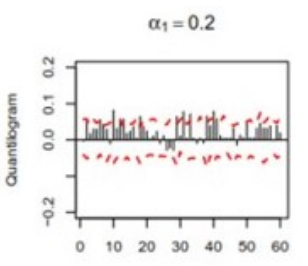

Lad

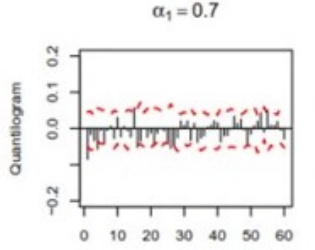

Lot

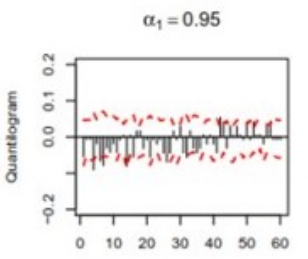

Lag

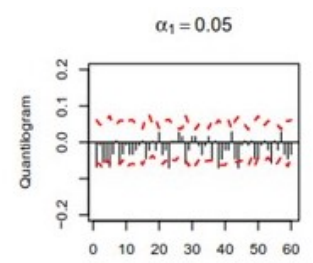

Lag

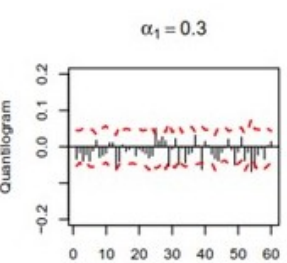

Lag

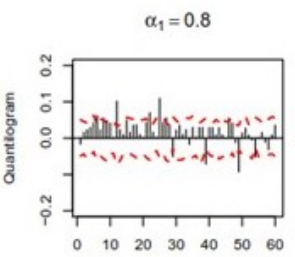

Log

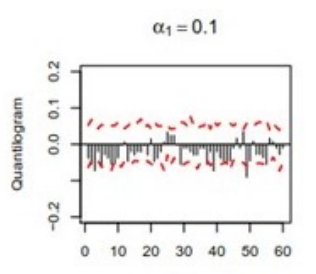

Lag

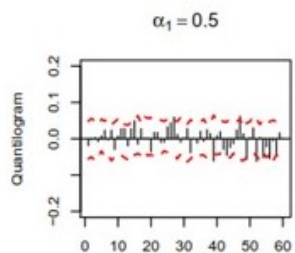

Lag

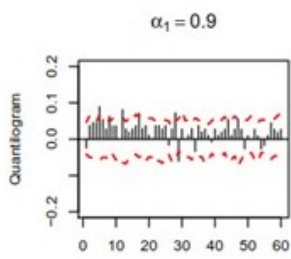

Lag

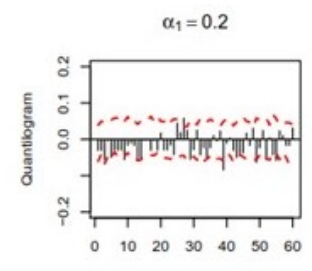

Lag

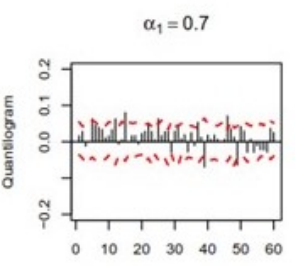

Las

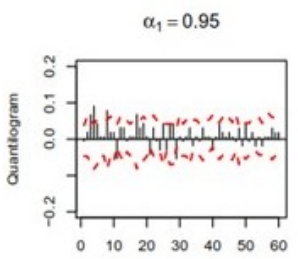

Lag

b) $\alpha_{2}=0.9$

Source: To detect directional predictability from NEM returns to Bitcoin returns, the sample cross quantilogram $\hat{p}_{\alpha}(k)$ for $\alpha_{2}=0.1$ and $\hat{p}_{\alpha}(k)$ for $\alpha_{2}=0.9$. The bar graphs explain sample cross quantilograms, and the lines are the $95 \%$ bootstrap confidence intervals. 
Figure 15: Cross-quantilogram representing directional predictability from Bitcoin returns to Gold returns

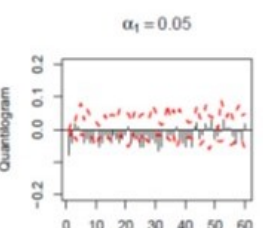

Las

$\boldsymbol{\alpha}_{1}=0.3$

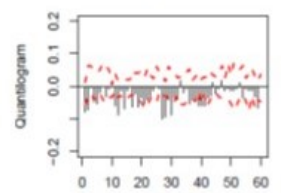

Lag

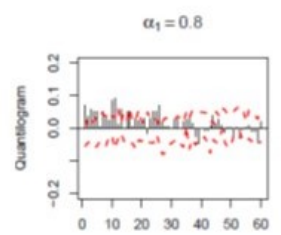

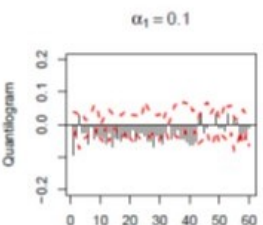

Las

$\boldsymbol{\alpha}_{1}=0.5$

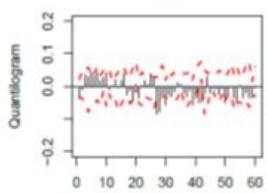

Las

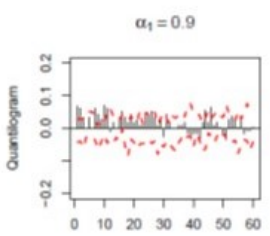

Lag

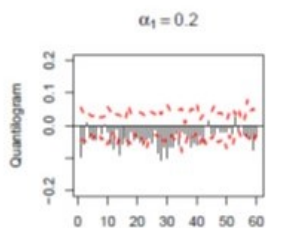

Los

$\alpha_{1}=0.7$

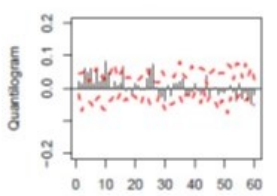

Lag

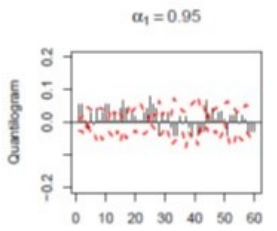

tas

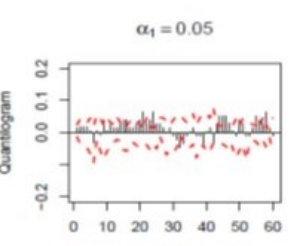

Lag

$\alpha_{1}=0.3$

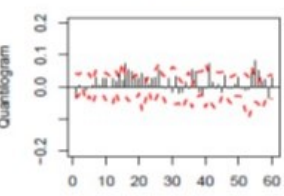

Lag

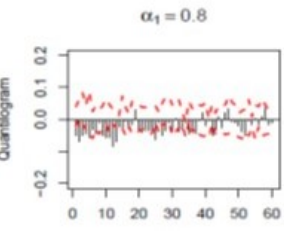

Lag

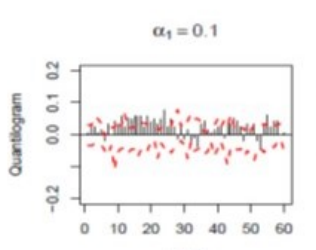

Lag

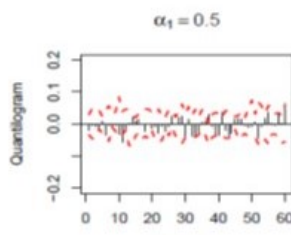

Lag

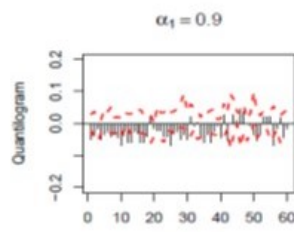

Lag

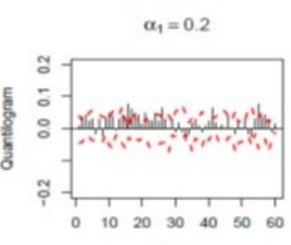

Lag

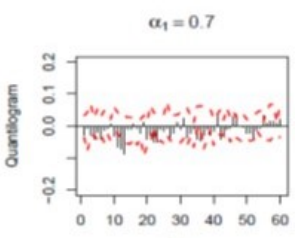

Lag

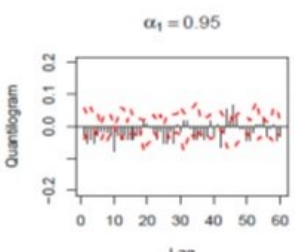

a) $\alpha_{2}=0.1$

b) $\alpha_{2}=0.9$

Source: To detect directional predictability from Bitcoin returns to Gold returns, the sample cross quantilogram $\hat{p}_{\alpha}(k)$ for $\alpha_{2}=0.1$ and $\hat{p}_{\alpha}(k)$ for $\alpha_{2}=0.9$ The bar graphs explain sample cross quantilograms, and the lines are the $95 \%$ bootstrap confidence intervals. 
Figure 16: Cross-quantilogram representing directional predictability from Bitcoin returns to Oil returns

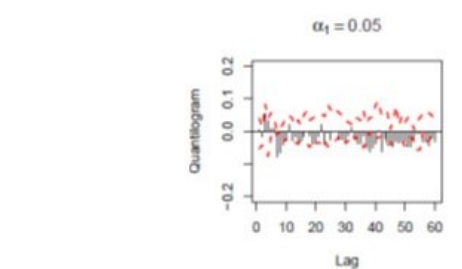

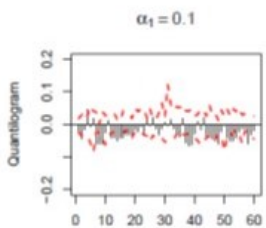

las

$\alpha_{1}=0.5$

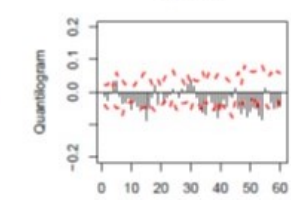

$\log$

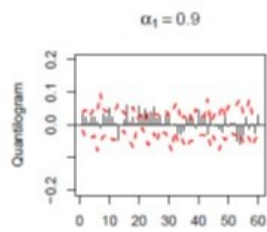

Los

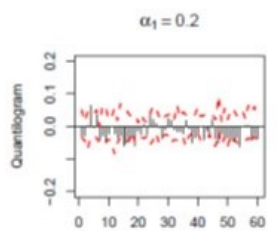

Lag
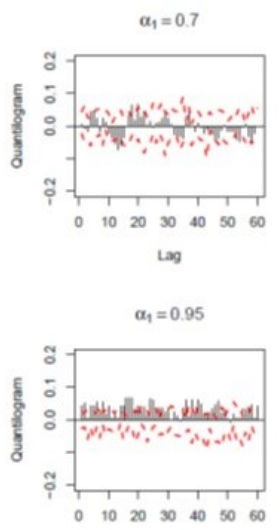

Lag

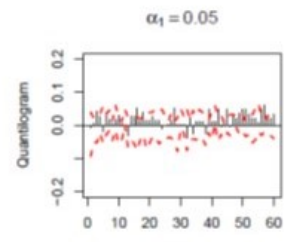

Lag

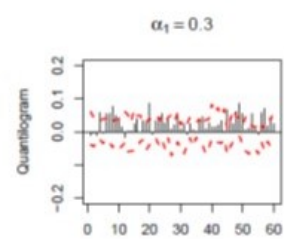

Lag

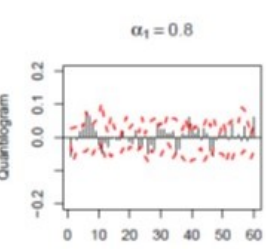

Lag

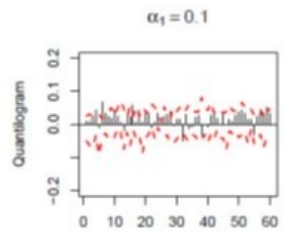

Lag

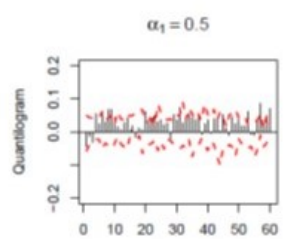

Lag

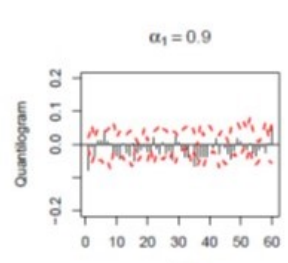

Lag

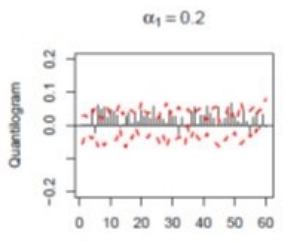

Lag

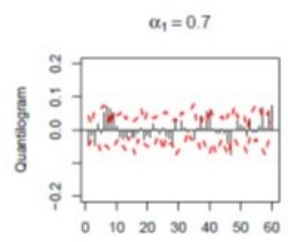

Lag

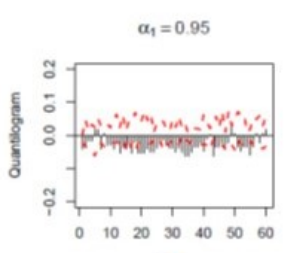

Lag

b) $\alpha_{2}=0.9$

Source: To detect directional predictability from Bitcoin returns to Oil returns, the sample cross quantilogram $\hat{p}_{\alpha}(k)$ for $\alpha_{2}=0.1$ and $\hat{p}_{\alpha}(k)$ for $\alpha_{2}=0.9$. The bar graphs explain sample cross quantilograms, and the lines are the $95 \%$ bootstrap confidence intervals. 
Figure 17: Cross-quantilogram representing directional predictability from Bitcoin returns to S\&P500 returns

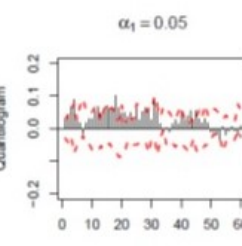

$\operatorname{lag}$

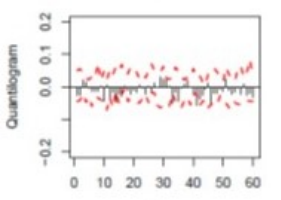

$\log$

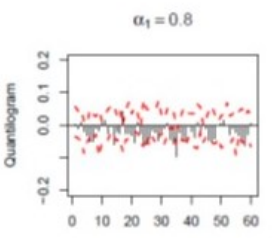

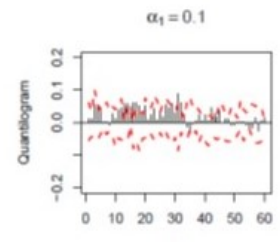

Lag

$\alpha_{1}=0.5$

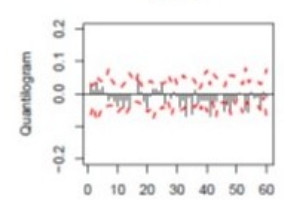

Las

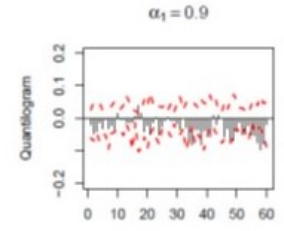

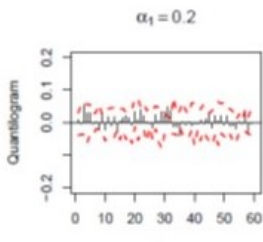

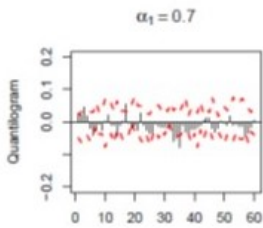

$\log$

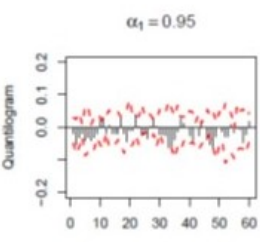

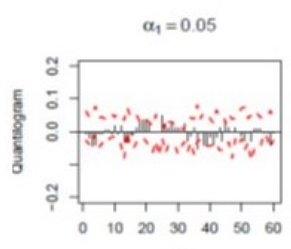

Lag
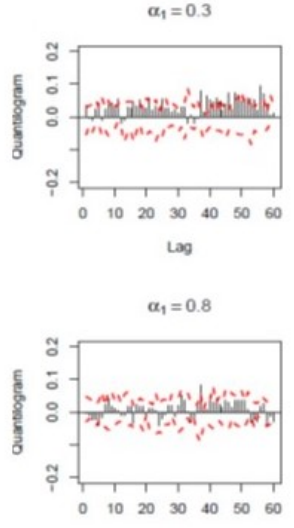
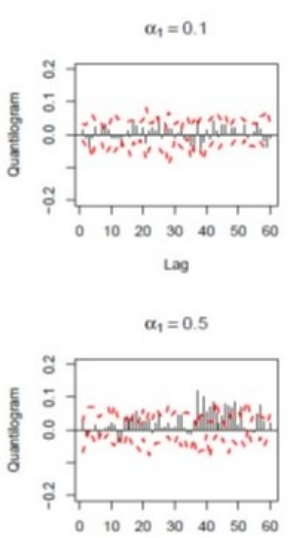

Lag

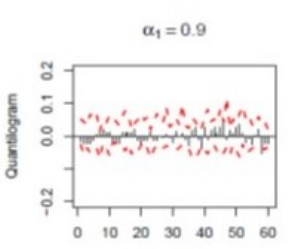

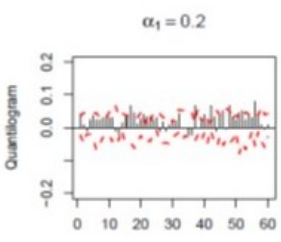
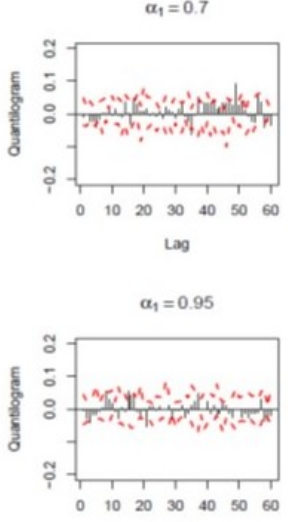

Las

Source: To detect directional predictability from Bitcoin returns to S\&P500 returns, the sample cross quantilogram $\hat{p}_{\alpha}(k)$ for $\alpha_{2}=0.1$ and $\hat{p}_{\alpha}(k)$ for $\alpha_{2}=0.9$ The bar graphs explain sample cross quantilograms, and the lines are the $95 \%$ bootstrap confidence intervals. 
Figure 18: Cross-quantilogram representing directional predictability from Gold returns to Bitcoin returns

$\alpha_{1}=0.05$

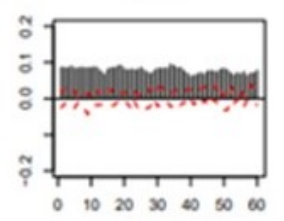

40

$\alpha_{1}=0.3$

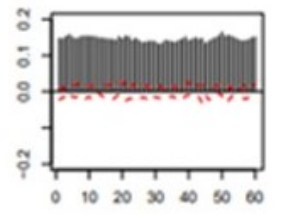

4

$\alpha_{1}=0.8$

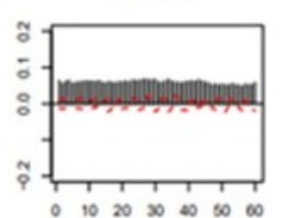

4 $\alpha_{1}=0.1$
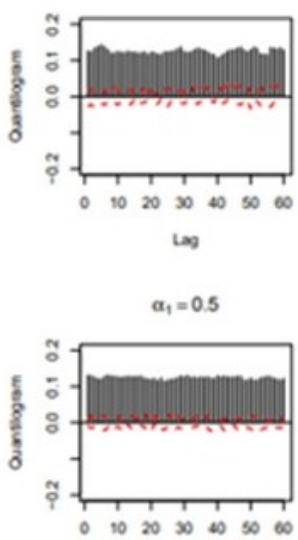

$1 \infty$

$\alpha_{1}=0.9$

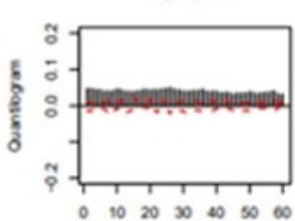

เ
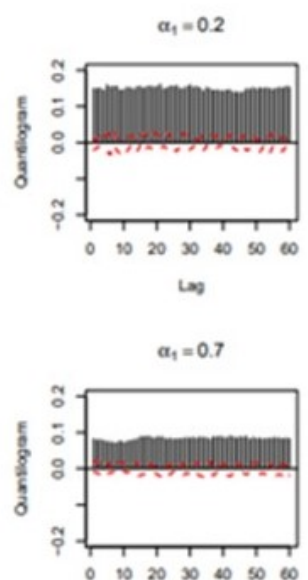

$\operatorname{La}$

$a_{1}=0.95$

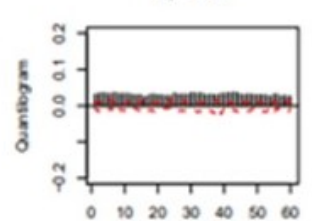

w

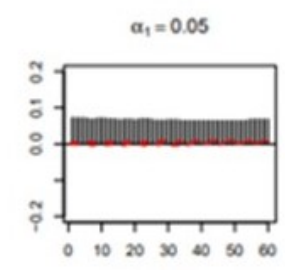

$\infty$

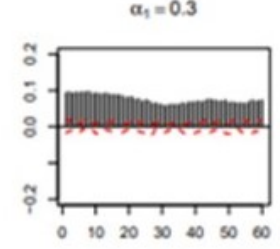

un

$a_{1}=0.8$

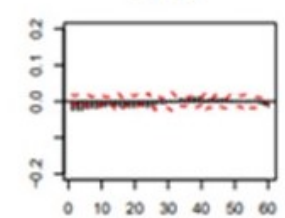

40

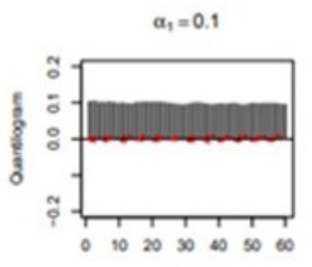

ᄂ

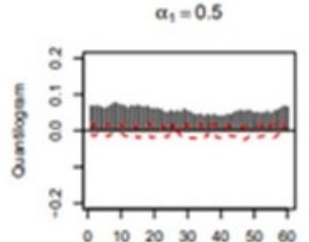

$\ln$

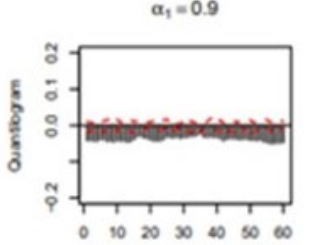

(⿻)

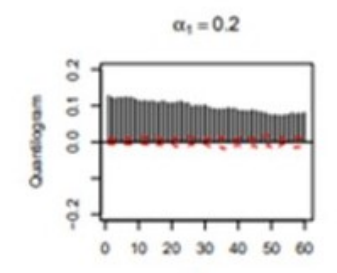

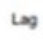

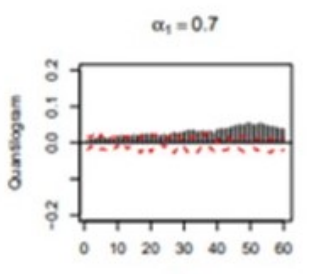

Los

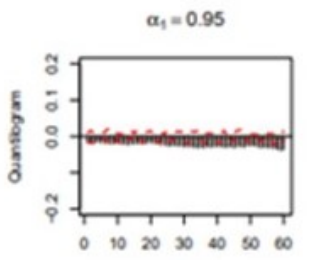

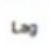

b) $\alpha_{2}=0.9$

Source: To detect directional predictability from Gold returns to Bitcoin returns, the sample cross quantilogram $\hat{p}_{\alpha}(k)$ for $\alpha_{2}=0.1$ and $\hat{p}_{\alpha}(k)$ for $\alpha_{2}=0.9$ The bar graphs explain sample cross quantilograms, and the lines are the $95 \%$ bootstrap confidence intervals. 
Figure 19: Cross-quantilogram representing directional predictability from Oil returns to Bitcoin returns

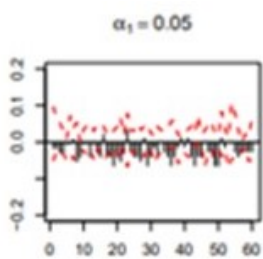

100

$\alpha_{1}=0.3$

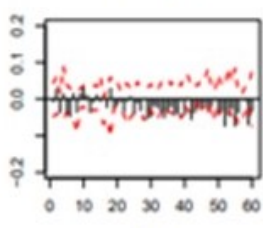

禹

$a_{1}=0.8$

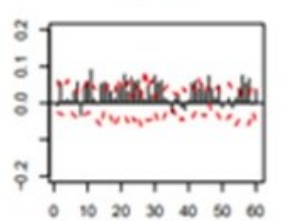

in

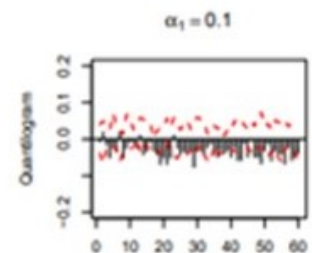

$\infty$

$a_{1}=0.5$

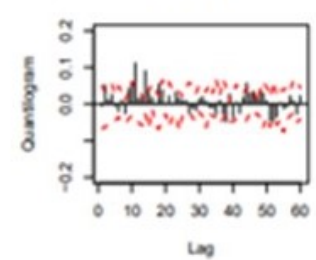

$\mathbf{a}_{1}=0.9$

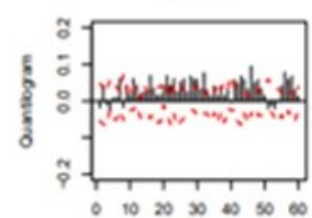

$\infty$

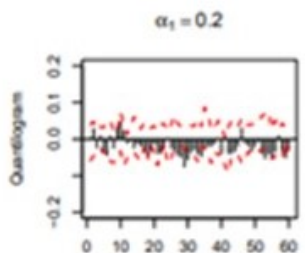

$\ln$

$\alpha_{4}=0.7$

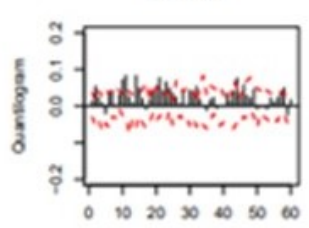

$\ln$

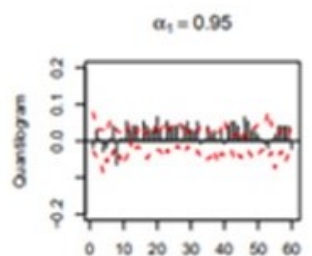

$\ln$

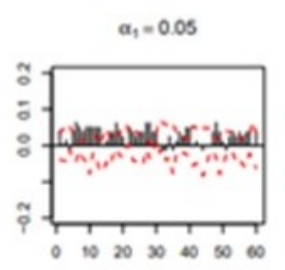

in

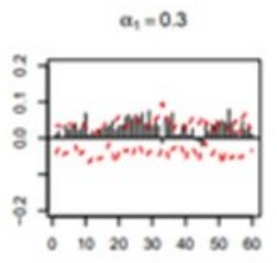

40

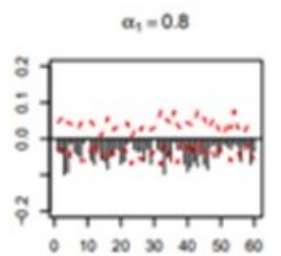

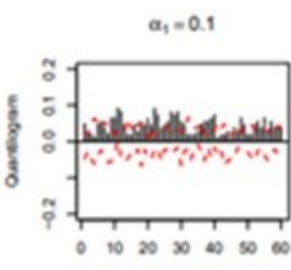

in

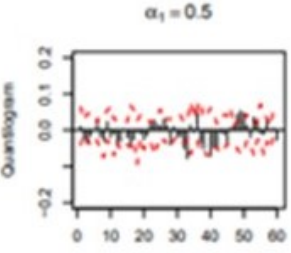

in

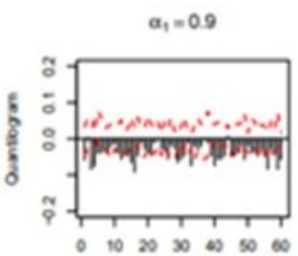

$\infty$

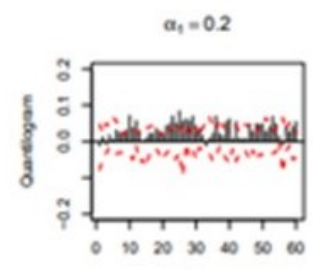

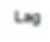

$a_{1}=0.7$
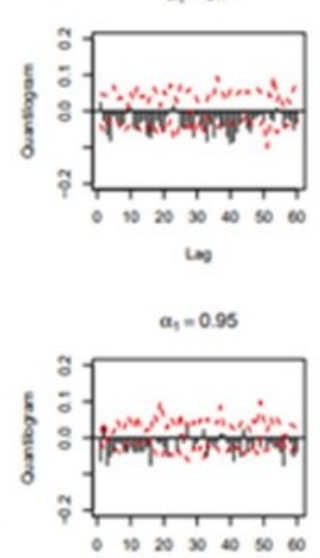

$m$

a) $\alpha_{2}=0.1$

b) $\alpha_{2}=0.9$

Source: To detect directional predictability from Oil returns to Bitcoin returns, the sample cross quantilogram $\hat{p}_{\alpha}(k)$ for $\alpha_{2}=0.1$ and $\hat{p}_{\alpha}(k)$ for $\alpha_{2}=0.9$. The bar graphs explain sample cross quantilograms, and the lines are the $95 \%$ bootstrap confidence intervals. 
Figure 20: Cross-quantilogram representing directional predictability from S\&P500 returns to Bitcoin returns

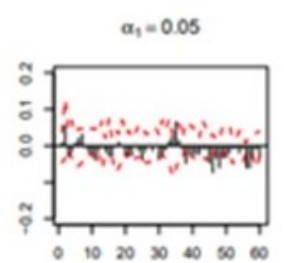

$\infty$

$\alpha_{1}=0.3$

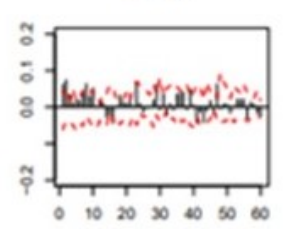

$\infty$

$\alpha_{1}=0.8$

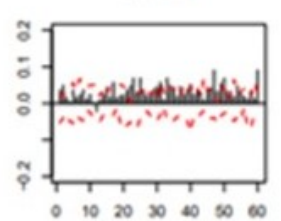

$\infty$

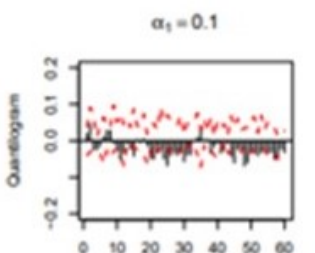

$1 \infty$

$\alpha_{1}=0.5$

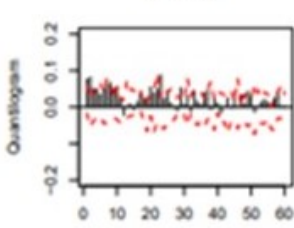

(⿻心)

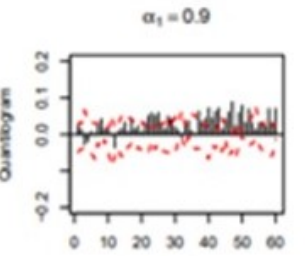

$\infty$

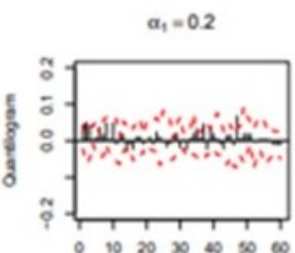

100

$\alpha_{1}=0.7$

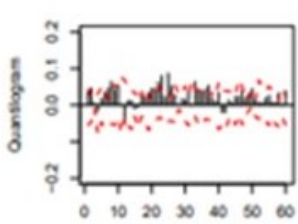

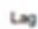

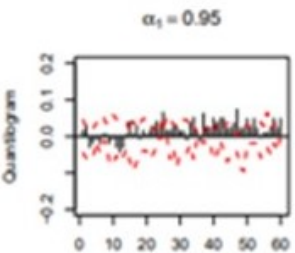

4

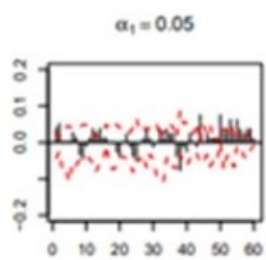

10

$\alpha_{1}=0.3$

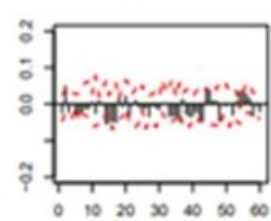

$\infty$

$a_{1}=0.8$

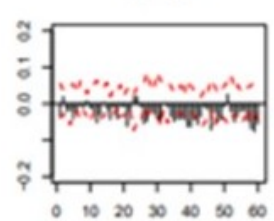

$\infty$

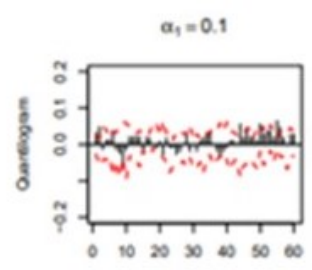

$\ln$

$a_{1}=0.5$

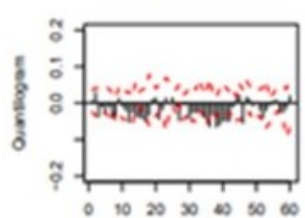

Li

$a_{1}=0.9$

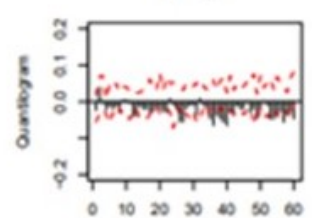

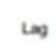

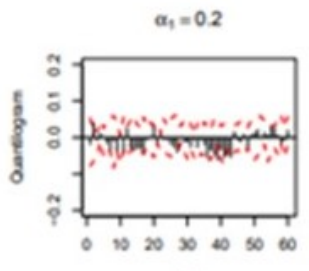

$\infty$

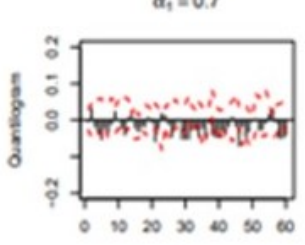

La

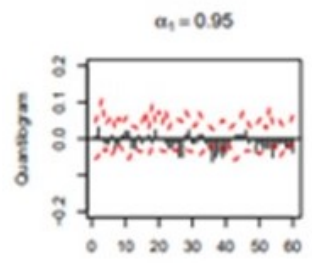

$\leftrightarrow$

$$
\text { a) } \alpha_{2}=0.1
$$

b) $\alpha_{2}=0.9$

Source: To detect directional predictability from Stock returns to Bitcoin returns, the sample cross quantilogram $\hat{p}_{\alpha}(k)$ for $\alpha_{2}=0.1$ and $\hat{p}_{\alpha}(k)$ for $\alpha_{2}=0.9$. The bar graphs explain sample cross quantilograms, and the lines are the $95 \%$ bootstrap confidence intervals. 
Table 1: Descriptive statistics

\begin{tabular}{lccccccccccc}
\hline & Bitcoin & Ripple & Ether & Stellar & Litecoin & Monero & Dash & NEM & Gold & Oil & S\&P500 \\
\hline Mean & 0.0020 & 0.0029 & 0.0030 & 0.0030 & 0.0016 & 0.0032 & 0.0024 & 0.0047 & 0.0002 & 0.0005 & 0.0003 \\
Median & 0.0025 & -0.0036 & -0.0010 & -0.0039 & -0.0004 & -0.0014 & -0.0014 & -0.0012 & 0.0002 & 0.0012 & 0.0003 \\
Maximum & 0.2252 & 1.0274 & 0.4123 & 0.7233 & 0.5104 & 0.5846 & 0.4378 & 0.9956 & 0.0480 & 0.1232 & 0.0496 \\
Minimum & -0.2075 & -0.6163 & -0.3021 & -0.3664 & -0.3952 & -0.2932 & -0.2432 & -0.3615 & -0.0333 & -0.0771 & -0.0410 \\
Std. Dev. & 0.0399 & 0.0753 & 0.0778 & 0.0831 & 0.0574 & 0.0708 & 0.0604 & 0.0886 & 0.0077 & 0.0233 & 0.0087 \\
Skewness & -0.2625 & 2.9956 & -3.3796 & 2.0363 & 1.2478 & 1.0068 & 0.8489 & 1.9764 & 0.4202 & 0.3606 & -0.4185 \\
Kurtosis & 7.6874 & 41.8764 & 67.5033 & 18.5068 & 15.4343 & 10.3197 & 8.9399 & 20.2051 & 3.0965 & 2.8078 & 4.4310 \\
\hline
\end{tabular}


Table 2: Testing for GCQ from Bitcoin to altcoins

\begin{tabular}{lccccccccccccc}
\hline & $1 \%$ & $5 \%$ & $10 \%$ & $20 \%$ & $30 \%$ & $40 \%$ & $50 \%$ & $60 \%$ & $70 \%$ & $80 \%$ & $90 \%$ & $95 \%$ & $99 \%$ \\
\hline Bitcoin to Ripple & 0.000 & 0.000 & 0.017 & 0.720 & 0.522 & 0.512 & 0.513 & 0.000 & 0.000 & 0.000 & 0.000 & 0.000 & 0.000 \\
Bitcoin to Ether & 0.000 & 0.000 & 0.040 & 0.835 & 0.468 & 0.476 & 0.504 & 0.000 & 0.000 & 0.000 & 0.000 & 0.000 & 0.000 \\
Bitcoin to Stellar & 0.000 & 0.000 & 0.001 & 0.501 & 0.487 & 0.510 & 0.496 & 0.000 & 0.000 & 0.000 & 0.000 & 0.000 & 0.000 \\
Bitcoin to Litecoin & 0.007 & 0.094 & 0.398 & 0.470 & 0.484 & 0.478 & 0.497 & 0.000 & 0.000 & 0.000 & 0.000 & 0.000 & 0.000 \\
Bitcoin to Monero & 0.000 & 0.000 & 0.051 & 0.794 & 0.487 & 0.496 & 0.485 & 0.000 & 0.000 & 0.000 & 0.000 & 0.000 & 0.000 \\
Bitcoin to Dash & 0.000 & 0.002 & 0.085 & 0.489 & 0.490 & 0.495 & 0.535 & 0.000 & 0.000 & 0.000 & 0.000 & 0.000 & 0.000 \\
Bitcoin to NEM & 0.000 & 0.001 & 0.035 & 0.755 & 0.504 & 0.515 & 0.500 & 0.000 & 0.000 & 0.000 & 0.000 & 0.000 & 0.000 \\
\hline
\end{tabular}

Note: We compute the quantile forecasts by inverting the parametric conditional copula distribution. We use six copulas (Gaussian, Frank, Clayton, Clayton Survival, Gumbel, and Gumbel Survival copulas). The check loss function is compared to evaluate the predictive ability of the different quantile forecasts from using the different copula models. The benchmark quantile forecasts are computed using the independent copula, so that there is no GCQ. Reported are the copula (which gives no GCQ). The to model GCQ and to make better quantile forecast. 
Table 3: Testing for GCQ from altcoins to Bitcoin

\begin{tabular}{|c|c|c|c|c|c|c|c|c|c|c|c|c|c|}
\hline & $1 \%$ & $5 \%$ & $10 \%$ & $20 \%$ & $30 \%$ & $40 \%$ & $50 \%$ & $60 \%$ & $70 \%$ & $80 \%$ & $90 \%$ & $95 \%$ & $99 \%$ \\
\hline Ripple to Bitcoin & 0.001 & 0.001 & 0.013 & 0.492 & 0.792 & 0.000 & 0.000 & 0.000 & 0.000 & 0.000 & 0.000 & 0.000 & 0.000 \\
\hline Ether to Bitcoin & 0.000 & 0.014 & 0.156 & 0.492 & 0.506 & 0.055 & 0.006 & 0.000 & 0.000 & 0.000 & 0.000 & 0.000 & 0.000 \\
\hline Stellar to Bitcoin & 0.000 & 0.000 & 0.007 & 0.650 & 0.850 & 0.335 & 0.007 & 0.000 & 0.000 & 0.000 & 0.000 & 0.000 & 0.000 \\
\hline Litecoin to Bitcoin & 0.000 & 0.003 & 0.016 & 0.020 & 0.004 & 0.027 & 0.472 & 0.000 & 0.000 & 0.000 & 0.000 & 0.000 & 0.000 \\
\hline Monero to Bitcoin & 0.000 & 0.010 & 0.316 & 0.486 & 0.220 & 0.234 & 0.006 & 0.000 & 0.000 & 0.000 & 0.000 & 0.000 & 0.000 \\
\hline Dash to Bitcoin & 0.000 & 0.007 & 0.108 & 0.481 & 0.477 & 0.128 & 0.101 & 0.000 & 0.000 & 0.000 & 0.000 & 0.000 & 0.000 \\
\hline NEM to Bitcoin & 0.000 & 0.003 & 0.072 & 0.788 & 0.480 & 0.074 & 0.052 & 0.000 & 0.000 & 0.000 & 0.000 & 0.000 & 0.000 \\
\hline
\end{tabular}


Table 4: Testing for GCQ from Bitcoin to gold, oil, and S\&P500, and vice versa

\begin{tabular}{lccccccccccccc}
\hline & $1 \%$ & $5 \%$ & $10 \%$ & $20 \%$ & $30 \%$ & $40 \%$ & $50 \%$ & $60 \%$ & $70 \%$ & $80 \%$ & $90 \%$ & $95 \%$ & $99 \%$ \\
\hline Bitcoin to Gold & 0.000 & 0.000 & 0.000 & 0.000 & 0.000 & 0.047 & 0.652 & 0.001 & 0.000 & 0.000 & 0.000 & 0.000 & 0.000 \\
Gold to Bitcoin & 0.000 & 0.000 & 0.000 & 0.000 & 0.001 & 0.027 & 0.576 & 0.003 & 0.000 & 0.000 & 0.000 & 0.000 & 0.000 \\
Bitcoin to Oil & 0.000 & 0.000 & 0.000 & 0.000 & 0.000 & 0.002 & 0.569 & 0.310 & 0.002 & 0.000 & 0.000 & 0.000 & 0.000 \\
Oil to Bitcoin & 0.000 & 0.000 & 0.000 & 0.000 & 0.000 & 0.000 & 0.721 & 0.000 & 0.000 & 0.000 & 0.000 & 0.000 & 0.000 \\
Bitcoin to S\&P500 & 0.000 & 0.000 & 0.000 & 0.440 & 0.534 & 0.006 & 0.218 & 0.182 & 0.061 & 0.226 & 0.000 & 0.000 & 0.000 \\
S\&P500 to Bitcoin & 0.000 & 0.000 & 0.000 & 0.047 & 0.051 & 0.001 & 0.554 & 0.029 & 0.047 & 0.084 & 0.000 & 0.000 & 0.000 \\
\hline
\end{tabular}

Note: We compute the quantile forecasts by inverting the parametric conditional copula distribution. We use six copulas (Gaussian, Frank, Clayton, Clayton Survival, Gumbel, and Gumbel Survival copulas). The check loss function is compared to evaluate the predictive ability of the different quantile forecasts from using the different copula models. The benchmark quantile forecasts are computed using the independent copula, so that there is no GCQ. Reported are the bootstrap p-values for testing the null hypothesis that none of these six copula models (which model GCQ) offers better quantile forecast than the independent copula (which gives no GCQ). The small p-values of the Reality Check indicate the rejection of the null hypothesis, suggesting that there exists a copula function to model GCQ and to make better quantile forecast. 


\section{Appendix}

Table A1: Granger causality in distribution (GCD) test results

\begin{tabular}{lc}
\hline \hline & Test statistic value \\
\hline Bitcoin to Ripple & $-64.74^{* * *}$ \\
Bitcoin to Ether & $-67.02^{* * *}$ \\
Bitcoin to Stellar & $-68.1^{* * *}$ \\
Bitcoin to Litecoin & $-82.81^{* * *}$ \\
Bitcoin to Monero & $-69.59^{* * *}$ \\
Bitcoin to Dash & $-66.49^{* * *}$ \\
Bitcoin to NEM & $-71.65^{* * *}$ \\
Bitcoin to Gold & $-64.49^{* * *}$ \\
Bitcoin to Oil & $-60.55^{* * *}$ \\
Bitcoin to S\&P & $-72.47^{* * *}$ \\
Ripple to Bitcoin & $-68.77^{* * *}$ \\
Ether to Bitcoin & $-66.19^{* * *}$ \\
Stellar to Bitcoin & $-69.19^{* * *}$ \\
Litecoin to to Bitcoin & $-55.58^{* * *}$ \\
Monero to Bitcoin & $-69.5^{* * *}$ \\
Dash to to Bitcoin & $-69.98^{* * *}$ \\
NEM to to Bitcoin & $-71.25^{* * *}$ \\
Gold to Bitcoin & $-80.24^{* * *}$ \\
Oil to Bitcoin & $-76.82^{* * *}$ \\
S\&P to Bitcoin & $-59.98^{* * *}$ \\
\hline \hline
\end{tabular}

Note: Reported are the Hong and Li [2004] test statistic values for testing the null hypothesis of non-GCD. The asymptotic p-values were calculated from the standard normal distribution. ${ }^{* * *}$ denotes significance at the $1 \%$ level. 